\title{
Non-contact multi-frequency magnetic induction spectroscopy system for industrial-scale bio-impedance measurement
}

\author{
M D O'Toole, L A Marsh, J L Davidson, Y M Tan, D W \\ Armitage, and A J Peyton \\ ${ }^{1}$ School of Electrical and Electronic Engineering, University of Manchester, \\ Manchester, M13 9PL, UK \\ E-mail: michael.otoole@manchester.ac.uk
}

\begin{abstract}
Biological tissues have a complex-impedance, or bio-impedance, profile which changes with respect to frequency. This is caused by dispersion mechanisms which govern how the electromagnetic field interacts with the tissue at the cellular and molecular level. Measuring the bio-impedance spectra of a biological sample can potentially provide insight into the sample's properties and its cellular structure. This has obvious applications in the medical, pharmaceutical and food-based industrial domains. However, measuring the bio-impedance spectra non-destructively and in a way which is practical at an industrial-scale presents substantial challenges. The low-conductivity of the sample requires a highly sensitive instrument, while the demands of industrialscale operation require a fast high-throughput sensor of rugged design.

In this paper, we describe a multi-frequency magnetic induction spectroscopy (MIS) system suitable for industrial-scale, non-contact, spectroscopic bioimpedance measurement over a bandwidth of $156 \mathrm{kHz}-2.5 \mathrm{MHz}$. The system sensitivity and performance are investigated using calibration and known reference samples. It is is shown to yield rapid and consistently sensitive results with good long-term stability. The system is then used to obtain conductivity spectra of a number biological test samples, including yeast suspensions of varying concentration and a range of agricultural produce, such as apples, pears, nectarines, kiwis, potatoes, oranges and tomatoes.
\end{abstract}

Keywords: magnetic induction, spectroscopy, bio-impedance, food industry, nondestructive testing.

Citation:

Meas. Sci. Technol. 26 (2015) 035102 (17pp).

DOI: 10.1088/0957-0233/26/3/035102 


\section{Introduction}

Bio-impedance spectroscopy is the measurement of the complex-impedance of a biological sample over a range of frequencies using time-varying electromagnetic fields. The purpose is to use the specifics of the measured spectra to obtain information on the physical, chemical, or cellular properties of the sample in a non-destructive way.

The impedance spectra of biological tissues are characterised by three main dispersions. Each dispersion is associated with a particular mechanism of interaction between the electromagnetic field and the cellular and molecular material which compose the sample. Gabriel et al. 1] summarises the dispersions thus:

- $\alpha$-dispersion: Operates at the lowest frequencies - hundreds of hertz. Caused by ionic diffusion processes at cellular membrane.

- $\beta$-dispersion: Operates in a frequency range between the other two dispersions - mainly the hundreds to megahertz range. Caused primarily by the polarisation of cell membranes blocking the flow of ions between the intra and extra cellular media. The cell membranes in this sense act as a form of capacitor.

- $\gamma$-dispersion: Operates at the highest frequencies - gigahertz region. Caused by the polarization of water molecules in the sample.

It follows that changes occurring in a biological test sample at the cellular or molecular level should, in principle, be reflected in the shape of the impedance spectra of that sample. For example, the characteristics of the $\beta$-dispersion are governed by the dielectric properties of the cell membrane. From this, we may assume that the shape or magnitude of the dispersion is indicative of the average integrity of the cell membranes, or the volume fraction within the sample of cells surrounded by cell membranes [2].

Impedance spectroscopy has been widely investigated as a means of non-destructive testing of biological samples in a range of applications. For example, it has been used extensively in the field of medical diagnostics and biological tissue imaging 3, 4, 5. In this paper, we restrict our scope to industrial-scale applications, which we interpret to mean relatively large-scale process industries with high-throughput, with specific focus on the food industry.
In these applications, speed and ease of measurement, combined with reliability and ruggedness, are the prime motivations for the design of the instrument. We focus specifically on the food industry, where bioimpedance spectroscopy is a popular field of enquiry and has shown much promise towards practical and commercial use.

\subsection{Bio-impedance spectroscopy in the food industry}

1.1.1. Meat inspection: The use of impedance spectroscopy to monitor and evaluate meat has been extensively explored, with the earliest experiments dating back as far as 1936 6]. Applications include the measurement of content, quality, and parameters associated with taste and acceptability to the consumer. Not all have shown positive results. Here, we examine some of the most promising:

One of the most prevalent areas of research using bio-impedance spectroscopy in meat-production is the early detection of two particular classifications of pork and beef products - pale, soft and exudative (PSE) meat and dark, firm and dry (DFD) meat. Both classifications are considered low-quality or defective meat grades which result in economic loss if they are not classified early enough. PSE meat has low $\mathrm{pH}$ and is strongly exudative resulting in considerable loss of juice [2]. DFD meat has high $\mathrm{pH}$ and is susceptible to spoiling [7. If detected sufficiently early, PSE and DFD carcasses can be assigned appropriately to mitigate the scale of economic loss. For instance, PSE meat can be used fresh, whereas DFD is suitable for sausages [2].

The factors which characterise PSE and DFD meats are related to the state of cellular breakdown and volume of extracellular fluid, and the condition of the muscle structure [8]. Consequently, we would expect to see abnormal changes to the $\beta$-dispersion over time in these types of meat when compared to normal meat, and that these changes might be a useful metric for prediction.

The most successful result of using bio-impedance spectra in this way was reported by Pliquett et al. [2]. Good correlations were found between $\mathrm{pH}$ and driploss three hours post-mortem, and the impedance ratio between upper-frequency and lower-frequency points of the dispersion. However, useful predictions so close to slaughter have not been replicated. Oliver et al. 9] had some success classifying normal pork from PSE after 
36 hours post-mortem, while more recently, CastroGiráldez et al. [8] observed significant differences between 24-48 hours post-mortem but none after only 12 hours.

Tenderness is one of the most important quality attributes of meat for the consumer 10 . Meat becomes more tender as it matures - caused by biochemical and physicochemical processes changing and breaking down the muscles in the meat 11]. The time required to reach a given tenderness is difficult to predict and can vary substantially between animals. In beef for example, optimal ageing times can range from two days to three weeks [12. Given this uncertainty over maturation times, there is need for a more scientific approach, which can monitor the tenderness of the meat as it ages and optimise the necessary storage and maturation time.

Bio-impedance spectroscopy has been identified as a possible method for assessing meat tenderness as it ages. The electrical properties of meat changes as it ages and its structure begins to alter and break down - particularly over the $\beta$ dispersion region where membrane integrity and porosity has the most influence on the shape of the spectra.

Byrne et al. [13] investigated the effects of ageing on heifers. Early post-mortem results were found to be poor, however, good correlations were obtained after two or more days ageing, when comparing highto-low frequency impedance ratios, with qualitative tenderness from taste tests and quantitatively, with the Warner-Bratzler shear forces exhibited by the meat. Similarly, Lepetit et al. 10 found linear relationships between impedance ratios and muscle fibre mechanical resistance. However, gradient changes between animals made this parameter alone unsuitable for predictive models.

More recent work has also used the degree of anisotropy in impedance across the muscle to evaluate the age and tenderness of the meat [10, 12]. Muscle tissue within the meat is composed of long conducting myofibres enclosed by endomysial envelopes which act as insulating membranes 12. The long and fibrous structure of the muscle leads to marked differences in impedance between measurements taken along the muscle fibres or transversely. The progressive breakdown of this structure leads to a reduction in this anisotropy. Damez et al. [12] used a system consisting of a circular ring of probes to return muscle impedance at different frequencies and in different directions. The different features were combined in linear models which correlated well with the results from mechanical compression stress tests.

The relative speed with which most impedance spectroscopy systems can obtain results makes this approach well suited to use in the feedback control sys- tems that typically inhabit processed-meat productionlines. For example, Chanet et al. [14 demonstrated impedance spectroscopy to measure and control the content of lipid and water in potted minced pork. Online monitoring in this case, precluded the need for a separation stage to obtain the proportion of the key ingredients, streamlining the manufacturing process and reducing potential for the introduction of contaminants.

More recently, Masot et al. 15 developed a system to monitor $\mathrm{NaCl}$ content in the process of salting minced pork loin. The amount of salt added to the meat during salting processes must be controlled, otherwise excessive salting can ruin the flavour of the meat and damage the health of the consumer. Masot et al. used a coaxial needle electrode to puncture the meat and obtain an impedance spectra of tissue inside the test sample. The system was able to predict the $\mathrm{NaCl}$ content in minced meat samples up to a concentration of $20 \%$ with good accuracy. The same system has also shown robust predictions of $\mathrm{NaCl}$ content, moisture, and water activity during the process of salmon salting [16].

1.1.2. Agricultural produce: The bio-impedance spectra of agricultural produce, such as fruit, vegetables, etc, may provide some insight into the state of cellular change, decay, or damage, and by relation, the physicochemical factors that can influence the quality factors important to the consumer. The ability to measure these attributes rapidly and non-destructively could be of considerable benefit [17, allowing for better quality assessment, and improved process control.

The ripening and maturation of fruit is a product of the structural and cellular changes occurring within. These changes should, in principle, be reflected in the shape of the $\beta$-dispersion, making the spectra a potentially useful indicator of the state and progress of these properties. There is some empirical evidence to support this notion. Changes in impedance have been observed during ripening of nectarines [18, persimmon fruit [19, garut citrus [20] and mangos 21] - particularly at the low-frequency end of the $\beta$ dispersion, which is most affected by cell membrane integrity. However, usable correlations and prediction models were not reported. Soltani et al. [22] reported ripeness level prediction for bananas using impedance results at $100 \mathrm{kHz}$. Good coefficients of determination were obtained although only a small number of samples were used. Juansah et al. 20] investigated the physiochemical properties associated with ripening in garut citrus fruit. This approach was more rigorous than simply observing the changes in the bio-impedance properties over time. Here the aim was to relate specific quantitative metrics 
to the impedance response. Good correlations were found between bio-impedance and $\mathrm{pH}$, Hydrogen ions, total soluble solids (TSS) and firmness. Thus far, the link between impedance spectra and the state of ripeness or maturation is only indicated rather than definitive. More robust correlations are required before bio-impedance spectra can be used as a metric of ripeness or maturation.

The identification of defects or damage in produce has been highlighted as a useful application particularly when the damage or defect cannot easily be detected by visible inspection. Chilling injury is a form of damage to fruit caused by storage at low temperatures. The injury results in the fruit ripening abnormally 23] giving it a dry and mealy taste. Harker \& Maindonald [24] and Harker \& Dunlop [18] explored the mechanism of chilling injury by observing the change in impedance spectra subject to different storage conditions. It was found that chilling injury inhibits some of the extracellular environment changes during ripening, evidenced by the different changes in impedance at low-frequencies between normal and chill-injured fruit. Similar, effects were also noted on the evolving impedance spectra of chill-injured persimmon fruit when compared with non-injured fruit [19.

The effect of apple-bruising on the impedance spectra has been investigated [25, 26]. The advantage of using impedance spectroscopy over visible methods to assess bruises is that the results are immediate there is no need to wait 1 day for tissue browning to develop as is typical protocol [26. Vozáry et al. 25] observed changes in the impedance ratio between high and low frequencies and bruising, where the pressing force causing the bruises was beyond the bio-yield point. Jackson \& Harker [26] found good correlations between bruise weight, and the change in low-frequency impedance before and after bruising. However, as the authors highlight, this was not a practical metric as impedance prior to bruising is unlikely to be known. Impedance ratios were not shown to be effective except where bruising was heavier. These results may be improved by different choice of upper frequency in the impedance ratio; the upper frequency in Jackson \& Harker appeared affected by the bruising. Ideally, for the impedance ratio to be effective, the higher frequency should be sufficiently high to be independent of changes in cellular structure.

1.1.3. Cell suspensions: Cell suspensions are structurally simpler and more homogenous than solid foods. This is advantageous for interpreting bio-impedance measurements as the number of processes occurring in the test sample are simplified and reduced. Further, there is a need for non-contact, fast and ro- bust methods to monitor and control cell suspensions during processes such as fermentation or coagulation. On-line monitoring and control has the potential to optimise yields and provide information for regulatory conformance. Properties of interest include cellconcentration and growth, cell-sizes and morphology, apoptosis, product quality, medium conditions, and metabolic state [27].

Carvell \& Dowd 28 and more recently Justice et al. 27. reviewed the use of bio-impedance spectroscopy as a method to monitor and process control in the bio-pharmaceutical industry. Promising results have been obtained for a range of cell cultures such as mammalian, plant and insect cells. Measurement of yeast biomass - or living cell volume - via impedance spectroscopy in bio-reactor fermentation processes has met with considerable success. Strong correlations have been shown repeatedly by different groups using impedance ratios 29, 30] and impedance differences 31] at dried weight concentrations as low as 0.3 $\mathrm{g} / \mathrm{l}$ [30. Further, commercial biomass monitors based on electrical impedance spectroscopy have now become available [32, 28. More directly applied to food processing, Kitamura et al. [33] showed good estimation of acidity and hardness in yogurt processing using impedance ratios. Li et al. 34 found impedance spectroscopy measurements useful in determining the end point of soya-milk coagulation when making tofu.

\subsection{Challenges of bio-impedance spectroscopy}

1.2.1. Selectivity: Selectivity is the ability to obtain good sensitivity to a single metric or signal, without being overly influenced by other properties in the test sample evolving at the same time. This is a particular challenge to the use of bio-impedance spectroscopy as a measurement technique. Biological samples are often complex entities with multiple processes which can influence the bulk impedance of the sample. Sometimes processes can be countervailing, masking the influence of one another from the spectra. In this case, predictive models using bio-impedance spectroscopy as an input may be impossible or unreliable.

The limits of selectivity have led to a number of surprising failures. Altman et al. 35 investigated the use of impedance spectroscopy to measure intramuscular fat in pork and beef samples. Despite finding clear evidence of variations of the impedance spectra with fat, good consistent correlations and predictive models could not be obtained. The authors conclude that the selectivity of the impedance method was not yet sufficient for practical use. Bauchot et al. 36. found no significant changes in impedance spectra of kiwifruit as it ripened. The authors suggested that the increased trans-membrane leakage of electrolytes 
in the kiwi, which should make it more conductive at lower frequencies, was countered by changes in the structure of the cell wall that bind the electrolytes, with the opposite effect on the conductivity. These countervailing influences cancel one another, resulting in insignificant changes to the spectra despite dramatic physical changes to the fruit.

Selectivity can be improved to some extent by incorporating a wider range of measurements and using more sophisticated means of extracting and connecting the relevant information. For instance, extending the bandwidth of the measurement, combined with physically relevant models, can help relate the shape of the spectra with the actual mechanisms and changes at the cellular level [37. The use of anisotropy measurement in evaluating meat tenderness is another example which has shown a degree of success - as discussed in section 1.1.1. Invariably the simplest and most robust approach however, is to choose applications where the properties of interest overwhelmingly influence the electrical spectral response of the sample.

1.2.2. Contact electrodes: The most direct method of impedance measurement is to use contact electrodes which touch or puncture the sample, then measure impedance between the electrodes. This method is used in most of the examples of impedance spectroscopy thus far described. However, the use of electrodes can be problematic.

There is a contact resistive impedance between the electrode and the sample which must be compensated for when taking measurements. At low-frequencies, a polarisation zone can form around the electrodes where the mobility of ions is different [12. This creates a contact capacitive impedance in addition to the contact resistive impedance. These combined impedances are a source of error and may substantially dominate the true bio-impedance of the sample. Chemical processes occurring within the sample itself may similarly alter electrode function. Harker \& Dunlop [18] found significant changes in the impedance of stainless steel electrodes due to the $\mathrm{pH}$ changes occurring in ripening fruit. These changes were found to be much larger than the actual impedance change occurring in the fruit. In some cases, issues surrounding contact impedance can be removed by use of tetra-polar or 4-point measurements [12] and by appropriate calibration.

The point of contact can be important when used on inhomogeneous samples. Different locations can give different results, and for consistency of measurements, the electrodes must be applied in the same place each time - something which is difficult to ensure in practice. Oliver et al. 9] found significant differences in correlations with instances of PSE and
DFD meat when the probe was tested on different parts of the meat. Only two out of the five regions tested gained positive correlations. Anisotropy in the test sample was a helpful quantifier in evaluating agerelated tenderness of meat, but in other cases, it can be a source of error and inconsistency. The operator not only has to ensure putting the electrodes in the right place, they also have to consider getting the right direction with respect to their previous measurements.

\subsection{Magnetic induction spectroscopy}

Magnetic induction spectroscopy (MIS) is a noncontact method of impedance measurement which uses induction rather than contact electrodes to obtain readings. The method works as follows: An excitation magnetic field is used to induce eddy currents across the sample at test. These currents generate a secondary magnetic field, which is then measured by a receiver coil. The impedance of the sample affects the magnitude and phase of the resulting secondary field, and can be calculated by comparing the excitation and secondary fields with respect to one another. Further, we can obtain an impedance spectra - rather than a single value - by using a multi-frequency excitation with components spread across the bandwidth of interest.

MIS is in general more challenging than direct electrical impedance. The required sensitivity is much higher in MIS and therefore, the design specification of the equipment is more demanding. However, the method is entirely non-contact, eliminating the need for electrodes and their accompanying problems. MIS is also well suited to the industrial environment. The constituent parts can be encased and protected from the harsh industrial environment, there are no moving or mechanical parts in the instrument, and it is capable of operating along high-throughput production lines. Single-frequency systems are already common place for detecting metal contaminants in the food industry. There is considerable potential for an MIS system to be used for bio-impedance spectroscopy on automated high-speed production lines provided that the system can be made sufficiently sensitive.

Recent work has begun to explore the possibility of using MIS in the food industry. Barai et al. 38. used an MIS system to investigate the conductivity spectra of various agricultural produce, including; potato, cucumber, tomato and banana, in addition to yeast suspensions of varying concentration. Clear $\beta$ dispersion responses were obtained over two frequency decades. However, the system was not designed for in-line industrial usage. It requires up to 52 seconds to obtain a measurement and therefore unsuitable for high-throughput processes

Euring et al. 39] used an adapted metal 
detector system to obtain single-frequency impedance measurements of apples. Using an existing metal detector design meant the system is already wellsuited to in-line operation on an industrial sortingline. Measuring only a single-frequency however, significantly diminishes the selectivity. Measurements were polluted by confounding variables such as test sample size and temperature. Measuring an extended bandwidth provides more information about the conductive processes on the cellular level. For instance, using spectroscopy means we can use impedance ratios at different frequencies to divide-out some of the confounding variables. More complex regression models can be used to improve correlations by incorporating the different frequency components into the analysis. Alternatively, model-fitting to lumped circuit models of the cellular structure can be used to infer more specific information about the internal state of the test sample.

In this paper, we present details of a magnetic induction spectroscopy system for rapid and robust non-contact measurement of the conductivity spectra of biological test samples over a frequency range from $156 \mathrm{kHz}$ to $2.5 \mathrm{MHz}$. The key features of the system are described which we have developed towards creating a highly-sensitive yet rugged and practical instrument suitable for industrial-scale use. The performance of the system is investigated using known test and calibration targets. Further, we demonstrate the applicability of the system to the food industry by obtaining conductivity spectra for yeast suspensions of varying concentrations and for a range of commonly available agricultural produce - such as apples, nectarines, tomatoes, potatoes, etc.

\section{Background Theory}

In a series of seminal papers, Gabriel et al. 1, 40, 41 reviewed and consolidated existing work on the dielectric measurements of biological tissue since the 1950s. The general shape of the spectral response for the different tissues are broadly the same. The conductivity $\sigma$ and relative permittivity $\epsilon$ are found to vary with frequency, $\sigma(\omega), \epsilon(\omega)$, for all biological spectra. The conductivity $\sigma$ increases and the relative permittivity decreases in a set of broad smooth steps over the frequency range. Each of the steps are associated with one of the three dispersions we have described. The step in the lowest frequency region is the $\alpha$-dispersion followed by the $\beta$ and $\gamma$ dispersions respectively. This is illustrated in figure 1. The review of literature presented thus far in this paper has continued to show this trend for the various different meat, agricultural products and cellular suspensions.

We only consider the $\beta$-dispersion in the present

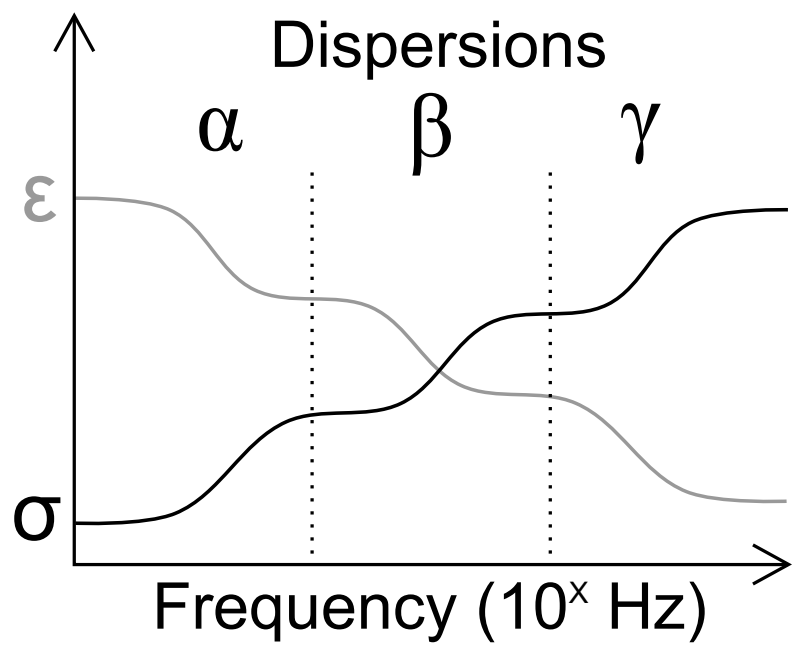

Figure 1. Conductivity and Permittivity trends in biological sample $-\alpha, \beta$ and $\gamma$ dispersions

work. Different models have been proposed to describe the behaviour of the spectra - ranging from the accurate but mostly empirical Cole-Cole model [41] to the more physically relevant models of Hayden et al. [42] and the more detailed version of Zhang et al. [43. The latter models relate individual elements of the cell to passive electrical components in a lumped circuit model. For example, the simplest model (Hayden et al.) models the cell as a resistor and capacitor in series. The resistor models the intracellular cytoplasmic resistance, and the capacitor models the cell membrane. The pair of components are connected in parallel with a second resistor which models the resistance of the extracellular fluid.

From an electrical engineering perspective, this gives us a highly intuitive model to explain the shape of the bio-impedance spectra - at least over the $\beta$ dispersion region. At low-frequencies, the capacitive cell-membrane presents high impedance and only the extracellular fluid resistance is dominant. At higher frequencies, the capacitive impedance diminishes, increasing the overall conductivity across the sample.

We now consider the case of how the dielectric properties can be obtained using magnetic induction. Define the dielectric properties of a material under test in terms of a complex relative permittivity:

$\epsilon^{*}=\epsilon_{r}-j \sigma /\left(\epsilon_{0} \omega\right)$

where $\epsilon_{r}$ is the relative permittivity of the material, $\sigma$ the total conductivity, $\omega$ the angular frequency and $\epsilon_{0}$ the permittivity of free-space.

Consider the scenario outlined in figure 2, Denote $\phi, \rho$ and $z$ as the rotational, radial and longitudinal component respectively of a cylindrical coordinate system. A non-magnetic dielectric sphere of radius $a$, positioned at the origin, is subject to a uniform timevarying magnetic field $\mathbf{B}=B_{z} e^{-j \omega t}$ operating along 


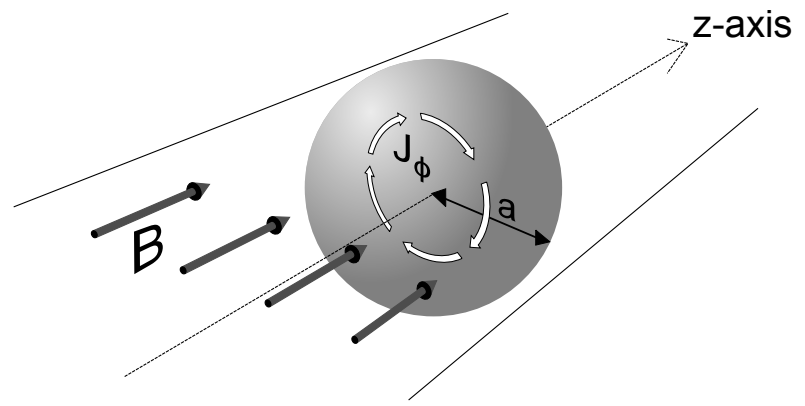

Figure 2. Eddy current problem in a spherical test sample

the $z$-axis. We refer to this as the excitation field. From Ampere's law, the magnetic field induces eddy currents $J_{\phi}$ in the sample about the $z$-axis.

It is easy to show using Maxwell's equations that $J_{\phi}$ is governed by the following differential equation,

$\nabla \times\left(\nabla \times J_{\phi}\right)+k^{2} J_{\phi}=0$

where $k$ is the propagation constant defined by,

$k^{2}=-\mu_{0} \epsilon_{0} \epsilon^{*} \omega^{2}$

and $\mu_{0}$ is the permeability of free space. The eddy currents $J_{\phi}$ in turn generate a secondary magnetic field and it is this secondary field which we are interested in measuring.

Bidinosti et al. 44 presents a solution for the magnetic field $\mathbf{B}^{\prime}$ at some point $p$ outside the sphere,

$B_{\rho}^{\prime}=\frac{3 B_{z}}{2} \frac{j_{2}(k a)}{j_{0}(k a)} \frac{a^{3} \rho z}{r^{5}}$

$B_{z}^{\prime}=B_{z}\left[1-\frac{j_{2}(k a)}{j_{0}(k a)} \frac{a^{3}\left(r^{2}-3 z^{2}\right)}{2 r^{5}}\right]$

where $j(\zeta)_{n}$ is a spherical Bessel function of the first kind and $r$ is the distance between the origin and point $p$ - i.e. $r^{2}=\rho^{2}+z^{2}$. We will use a receive coil geometry which tangentially intersects the $z$-axis of the secondary field. Only the flux in the z-direction will be enclosed by the coil, thus only $B_{z}$ is measured.

The spherical Bessel functions $j_{2}(\zeta) / j_{0}(\zeta)$ can be expanded about $\zeta=0$ (see Bidinosti et al. - appendix). Taking only the first component of the expansion,

$\frac{j_{2}(\zeta)}{j_{0}(\zeta)} \approx \frac{\zeta^{2}}{15}$

equation (4) can be simplified to,

$B_{z}^{\prime}=B_{z}+P a^{5} \mu_{0} \epsilon_{0} \epsilon^{*} \omega^{2} B_{z}$

where $P=\left(r^{2}-3 z^{2}\right) /\left(30 r^{5}\right)$. $P$ can be considered more broadly as a geometric constant unique to some specific coil geometry.

Denote $\Delta B=B_{z}^{\prime}-B_{z}$ as the secondary field emitted solely by the object, that is, where the influence of the excitation has been removed. Then, substituting for $\epsilon^{*}$ we have,

$\frac{\Delta B}{B_{z}}=P a^{5} \mu_{0} \omega\left(\epsilon_{0} \epsilon_{r} \omega-j \sigma\right)$.
Clearly, conductivity is proportional to the imaginary component of the measured signal, and permittivity proportional to the real component.

Finally, we introduce a term which will be useful in interpreting our results. Relative conductivity $\Sigma$ we define as the imaginary component of equation (7) divided by the angular frequency $\omega$,

$\Sigma=P a^{5} \mu_{0} \sigma$.

\section{Multi-Frequency Magnetic Induction System}

The multi-frequency magnetic induction system obtains a five-point impedance spectra of a sample from a single-pass. The system consists of a mechanical sensor-head containing the induction coil assembly, transmit hardware to drive the excitation coil, receive hardware to detect the secondary magnetic field induced by the eddy-currents in the sample, and an ADC/DAC-FPGA system for signal processing and waveform generation. In addition, the system also consists of a host-PC to interface with the operator, and a conveyor assembly to convey the samples through the aperture of the sensor-head. A schematic of the system is shown in figure 3 and the system and its components are shown in figure 4 .

The system operates in the following way: A multi-frequency waveform is generated by the ADC/DAC-FPGA system and input to the transmit hardware. The transmit hardware drives a transmit coil in the sensor-head with high current. This generates an excitation magnetic field in the aperture of the sensor-head operating at the same frequencies as the input multi-frequency waveform.

A sample is placed on a carriage on the conveyor assembly and is passed through the aperture of the sensor-head. The excitation field induces eddycurrents in the sample as it passes, which in turn generate a secondary magnetic field. This secondary field is detected by the receive coil assembly and amplified using the receive hardware. The signal is returned to the $\mathrm{ADC} / \mathrm{DAC}-\mathrm{FPGA}$ system where it is processed and the significant frequency components corresponding to the multi-frequency input waveform are extracted to obtain a spectra of the sample from a single-pass. The system is currently set to be able to process samples approximately every 1.5 seconds.

\subsection{Sensor-head}

A plan of the sensor-head is shown in figure 3 . It consists of an arched-shaped aperture of $160 \mathrm{~mm}$ width and $290 \mathrm{~mm}$ height (outer-edge dimension) running through the centre of the head and enclosed in an archshaped aluminium shell $0.36 \mathrm{~m} \times 0.49 \mathrm{~m} \times 0.34 \mathrm{~m}(\mathrm{w}$ 


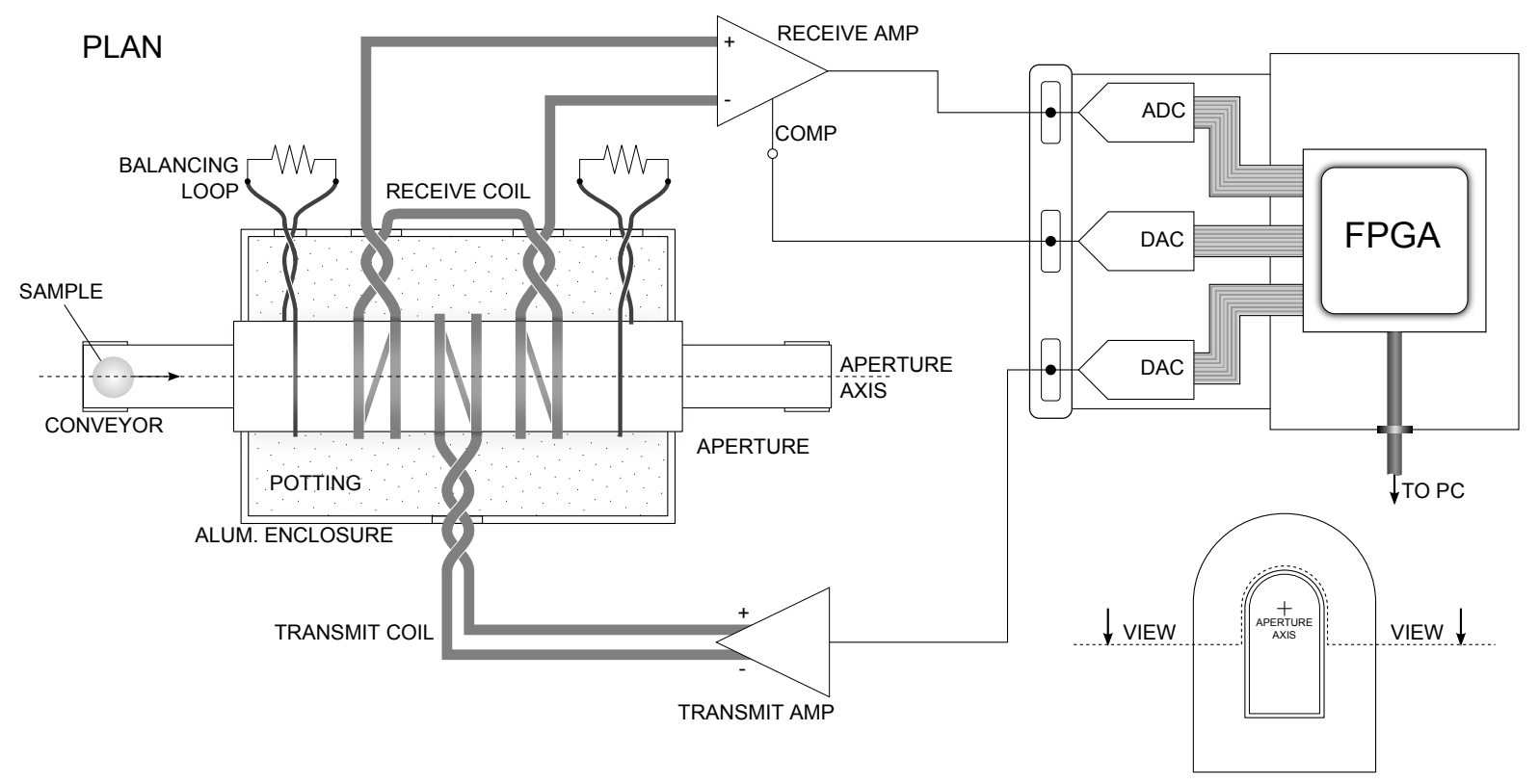

Figure 3. Magnetic induction spectroscopy system schematic

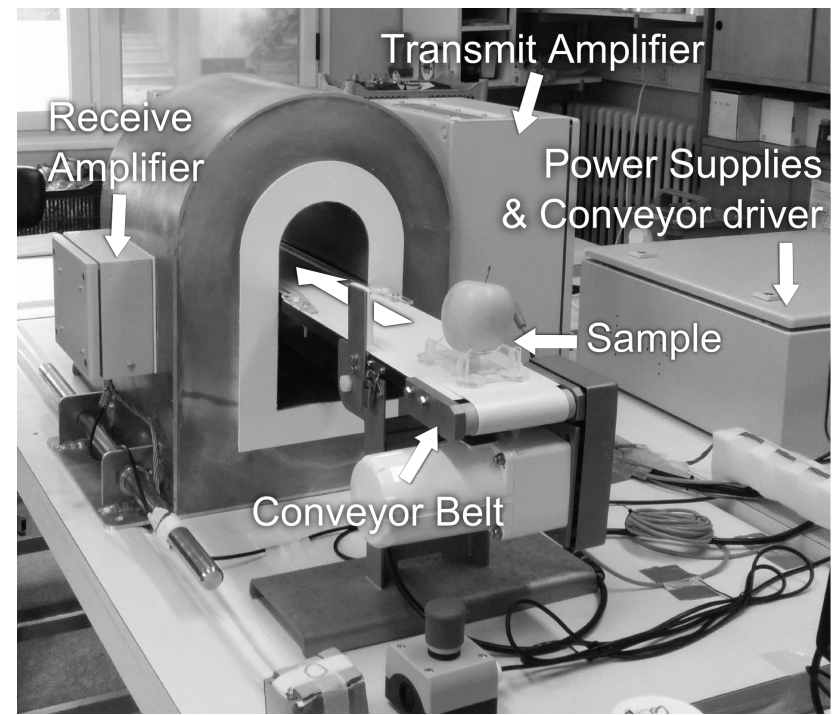

Figure 4. Multi-Frequency Magnetic Induction Spectroscopy System

$\times \mathrm{h} \times \mathrm{l})$. The transmit and receive coils are wound around the arched-shaped aperture in a gradiometer arrangement, with the transmit coil wrapped around the centre and the two receive coils wrapped $60 \mathrm{~mm}$ on either side of the transmit coil. The coils have dimensions $160 \mathrm{~mm}$ width and $290 \mathrm{~mm}$ height, and have two turns each. The resonant frequencies of the coils are well-away from the frequency range of interest.

The samples are positioned to pass through the radial centre of the archway - the 'aperture-axis' in figure 3 The arch-shape is chosen so that the coils are as close to the sample as possible while leaving room for a conveyor system beneath the sample typical of a sorting-line in an industrial setting.

The receive coils are connected in opposing directions such that emf generated by the background excitation field in one receive coil will be cancelled by the emf generated in the second receive coil by the same excitation field. When the system is inbalance, only the magnetic field induced by the sample will be detected. However, achieving this state of balance is difficult. In practice, small asymmetries in manufacture imbalance the gradiometer, resulting in some, but not all, of the excitation field being cancelled.

Two additional single-turn coils, referred to as balancing coils, are wrapped around each end of the aperture. These are used to manually adjust the balance of the gradiometer. The coils are placed at the distal ends of the archway approximately $20 \mathrm{~mm}$ outwards from each receive coil. These coils are each connected to a potentiometer used to change the coil resistance. The coils generate their own secondary magnetic field in response to the excitation field, which interacts with the excitation field in the receive coil. The magnitude of this secondary field is dependent on the coil resistance set by the potentiometer. Assuming that the secondary field is close in phase to the excitation field, e.g. because the frequency is relatively low, or that the inductance of the balancing coil matches the transmit coil, then the coil resistance can be adjusted such that the residual uncancelled emf from the excitation field in the receive coil is cancelled by the emf induced by the secondary field of the balancing coils. 
The internal walls of the archway are lined with a conductive graphite paint (Safeline Metal detection systems, UK) to act as an electrostatic screen, mitigating capacitive coupling between the test sample and the receive coils. The graphite paint was repeatedly layered onto the internal walls to reach a resistivity of approximately $100 \Omega$ per square as per the manufacturers recommendation. Phase shifts caused by the screen are compensated for by the calibration procedure which we later describe in section 4.4 .

A PVC liner is used to protect the screen. The sensor-head is enclosed in an aluminium shell for structural rigidity and EMI screening. The space between the archway and the inside of the aluminium shell is filled with a potting resin to fix the coil positions in place with respect to the rest of the assembly and provide further structure to the head.

The mechanical design of the head was subject to extensive simulations using Maxwell electromagnetic field simulation software (Ansys, USA) to optimise the coil dimensions, coil spacings and aluminium enclosure dimensions. The solution maximises sensitivity to a $80 \mathrm{~mm}$ diameter spherical object passing through the radial centre of the archway, given the size constraints imposed by the need for a conveyor or sorting-line to fit through the aperture, and for its practical deployment and use in a laboratory or industrial setting

\subsection{Transmit}

The transmit coil is driven by a pair of amplifier-blocks mounted in the large side-case of the sensor head, as shown in figure 4. The amplifier-blocks can each source up to $10 \mathrm{~A}$ with an output voltage of approximately $32 \mathrm{~V}$ pk up to $7 \mathrm{MHz}$ cut-off frequency.

The transmit signal is generated by the analogue output of the ADC/DAC-FPGA system and input to the amplifier-blocks. The transmit signal is a multi-frequency waveform consisting of five frequency components with amplitudes for each component proportioned according to $1 / \sqrt{f}$ ratio. This relation was found by experiment to offer a good compromise between sensitivity at high and low frequencies. The current across the transmit coil at each frequency component is shown in figure 5

\subsection{Receive}

The receive side electronics consist of a transmissionline-transformer and a custom high-gain differentialamplifier block. The amplifier has a bandwidth of 10 $\mathrm{kHz}$ to $10 \mathrm{MHz}$, a gain of approximately $90 \mathrm{~dB}$, and an output-referenced input-noise of approximately 11.9 $\mathrm{nV} / \sqrt{H z}$.

The amplifier has an additional port (COMP in figure 3) on the input-side for a compensation

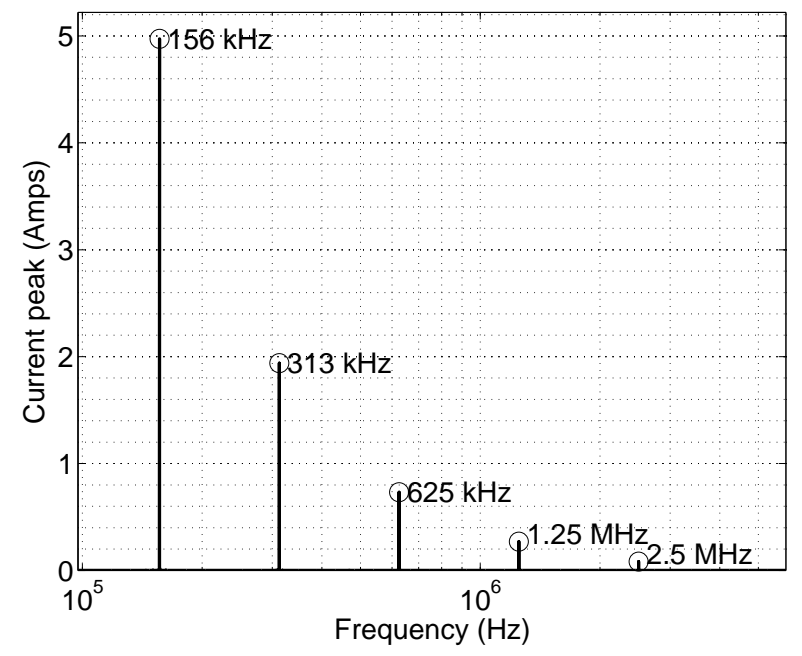

Figure 5. Approximate current at each frequency component of the transmit waveform

signal used to further cancel the imbalance of the gradiometer. The practical limitations of mechanical balancing on such a high-gain amplifier result in saturation at the output, even with the balancing coils deployed. By injecting a signal to compensate for the coil imbalance in the first-stage of the amplifier, we can retain the output within the amplifier's linear region.

\subsection{FPGA-based signal processing}

Signal processing is performed on a ADC/DAC-FPGA system (NI PXIe-7966R FlexRIO FPGA module with NI 5781 baseband transceiver adapter module, National Instruments, USA), and host PC linked to the ADC/DAC-FPGA system. The ADC/DAC module consists of two 14-bit differential analogue-todigital conversion ports and two 16-bit analogue output ports that both operate at a sampling rate of $100 \mathrm{MS} / \mathrm{s}$. The FPGA is driven by a $40 \mathrm{MHz}$ clock.

The ADC/DAC-FPGA system has three main functions; 1) to output the multi-frequency transmit waveform shown, 2) to output the compensation waveform for the auto-nulling algorithm described in the following sub-section, and 3) to filter the incoming signal from the receive amplifier and return the frequency components to the host PC.

The compensation signal is injected into the receive amplifier to cancel or null any residual excitation signal not already cancelled by the gradiometer and balance coils. The compensation waveform is computed by the host PC using the algorithm described in the next section, then loaded onto the FPGA module.

The receive signals are filtered on the FPGA using a block-averaging method. A block of data is stored on the FPGA. The next block is then averaged elementwise with this block, i.e. the first element of the first 
block is averaged with the first element of the second block and so on. The new averaged block is then stored. This is repeated until a predefined number of blocks have been averaged.

The block averaging method is optimal for reducing non-coherent noise in the signal. We are only interested in frequency components which are coherent as we choose an integer number of cycles for the transmit waveform and output it with the same clock as the receive. Similarly, the input receive and the compensation signal operate on the same clock and are coherent. The averaged block is demodulated on the FPGA using a fast Fourier transform (FFT) and the resulting components passed to the host program.

We set the size of each block to be 4096 elements long and set the number of blocks to be averaged together before demodulation by FFT as 100. For an FPGA driven at $40 \mathrm{MHz}$, the resulting rate at which the receive signal frequency components are returned to the host program is approximately $96.8 \mathrm{~Hz}(40$ $\mathrm{MHz} /(100 \times 4096))$.

The host program interfaces with the FPGA computing and loading the output waveforms and receiving the input waveforms. In addition, the host program provides the control interface for the operator, saves the input data, controls the conveyor-belt and photo-diode logic, and monitors the temperature of the transmit-amplifier heat sinks.

\subsection{Auto-nulling of background signal}

The background excitation signal is mostly cancelled in the receive coil by the gradiometer arrangement of the coil assembly, and by the passive electrical affect of the balancing loops. However, some residual signal remains which can dominate the secondary field we are trying to measure, and can saturate the receive amplifier.

A compensation waveform is used to cancel the remaining background signal. The waveform is computed on the host PC using the frequency components of the last input receive signal, then passed to the ADC/DAC-FPGA system which injects the waveform in to the receive amplifier. The summation of the two signals in the amplifier results in their cancellation leaving the output of the amplifier unsaturated and free of the residual background signal.

This forms a feedback loop: receive signal frequency components used to calculate new compensation waveform, new compensation waveform to ADC/DAC-FPGA, DAC output to receive amplifier, new receive signal, etc. The loop updates every time a new set of receive frequency components are returned from the ADC/DAC-FPGA to the host PC. Thus, the compensation waveform is updated at the same rate $(96.8 \mathrm{~Hz})$.
Using a feedback loop in this way means we can also null out drifting imbalances, e.g. due to thermal influences, harmonic distortions and unwanted environmental but coherent frequency noise. This method is simple and effective and can be implemented on relatively modest low-cost hardware. The latter is especially advantageous given our aim of producing a system suitable for industrial use.

A simple first-order update-law is used to compute the compensation waveform. Denote $X_{n} \in \mathbb{C}^{N}$ as a vector of $N$ elements containing the frequency components of the receive signal returned by the FPGA module. The subscript $n$ indicates that it is the $n^{\text {th }}$ block of components to be returned. The frequency components of a new compensation waveform $Y_{n+1} \in$ $\mathbb{C}^{N}$ are computed using the following,

$Y_{n+1}=Y_{n}+\gamma\left(H(\omega) \star R(\theta) \star X_{n}\right)$

$y_{n+1}=\mathcal{F}^{-1}\left(Y_{n+1}\right)$

where $\star$ indicates element-wise vector multiplication, $\gamma \in(0,1)$ is a scalar gain term chosen such that $Y$ is stable, $H \in \mathbb{R}^{N}$ is the frequency-domain kernel of a low-pass filter, and $R \in \mathbb{C}^{N}$ is a vector used to compensate for phase-shift from the receive amplifier and time-delays in the ADC/DAC module. The lowpass filter kernel $H$ is used to suppress higher frequency components which are difficult to null. We found a filter kernel with cut-off frequency around $5 \mathrm{MHz}$ sufficient for this purpose.

The function $\mathcal{F}^{-1}$ is the inverse Fourier transform, and $y_{n+1}$ the resulting time-domain representation of the new compensation signal. The host PC returns $y_{n+1}$ to the FPGA which then outputs this waveform on the compensation analogue output.

The gain term $\gamma$ is chosen by trial-and-error. Clearly, as $\gamma \rightarrow 0$ the stability of the algorithm improves at the expense of longer settling time. A suitable gain-term can be chosen by simply observing the effect of the gain-term on the receive signal. If high-frequency oscillations dominate and saturate the amplifier then the gain-term needs to be reduced. From experience, the algorithm implemented on the hardware described was found to be relatively robust, and finding suitable values of $\gamma$ was a straightforward task. When in operation, the gain-term can be switched to zero to suppress the auto-nulling and prevent the nulling of emf induced by the test sample.

\section{Method}

\subsection{Preparation of test samples}

4.1.1. Saline solutions: A set of saline solutions were used as constant conductivity targets for validating the system response. The solutions were prepared using salt $(\mathrm{NaCl})$ and deionised water with the conductivity 
measured using a combined conductivity probe and temperature sensor (4510 conductivity meter with 027013 glass-bodied probe, Jenway, UK). Two sets of solutions were created: The first set were made using $300 \mathrm{ml}$ glass jars with internal dimensions of $65 \mathrm{~mm}$ diameter by $80 \mathrm{~mm}$ length. The jars were filled to the brim with the saline solution to remove all air pockets and then sealed shut. The second set of solutions were prepared in $57 \mathrm{~mm}$ diameter thin PVC spheres. The solutions were re-tested for conductivity directly after use to ensure the conductivity remained stable.

4.1.2. Food samples: A range of agricultural products were scanned using the system to obtain their conductivity spectra. Three samples of each type or variety were tested. These were designated A, B and C. The characteristics of each test sample are shown in table 1. The parameter $\mathrm{h}$ is the height of the sample, $\mathrm{D}_{1}$ is the width at the widest point of the sample along a plane normal to the height measurement, and $\mathrm{D}_{2}$ is the width along an axis normal to $D_{1}$ and $h$. When scanned, the sample is positioned such that the axis along which $D_{1}$ is measured (the widest point of the sample) is placed across the entrance of the aperture. That is, the widest face of the sample faces the aperture. The exceptions are the kiwi, potato and pear, where the shortest width $\left(\mathrm{D}_{2}\right)$ is placed across the entrance to the aperture. The pears are rotated backwards such that the pointed-end faces directly away from the direction of travel. The volumes of the samples are obtained from displaced water when the sample is submerged.

The temperature of the sample is taken at the completion of each set of scans. Temperature is taken using a temperature probe (72-7715 Thermometer, Tenma) inserted $40 \mathrm{~mm}$ into the sample at its widest point in the direction of the core. Note that as the temperature measurement is destructive, the samples were not used again afterward. The samples are not subject to any physical modification or processing prior to scanning and are left entirely intact.

4.1.3. Yeast suspensions: Yeast suspensions were prepared by adding dried yeast (Saccharomyces cerevisiae) to deionised water held at a temperature between $30.0-30.8{ }^{\circ} \mathrm{C}$. An initial suspension of $60 \mathrm{~g}$ dried yeast to $300 \mathrm{ml}$ deionised water was created $(200$ $\mathrm{g} / \mathrm{l}$ ) followed by concentrations of $100 \mathrm{~g} / \mathrm{l}, 50 \mathrm{~g} / \mathrm{l}$ and 25 $\mathrm{g} / \mathrm{l}$ made by successive dilutions. The suspensions are contained in $300 \mathrm{ml}$ glass jars (see 'Saline Solutions') filled to the brim to remove air pockets, and sealedshut.

\subsection{Scan procedure}

The system is engaged and allowed to run for several minutes prior to scanning to ensure it has reached a steady operating temperature. The conveyor-belt system is activated and set to a speed of approximately $250 \mathrm{~mm} / \mathrm{sec}$. The system is set to auto-null until any remaining excitation signal components are removed (nulled) and the measured signal from the receive amplifier reaches some defined rms threshold.

To run a scan, the sample is first placed in a caddy and dropped on to one end of the moving conveyorbelt. The sample passes through a photo-diode which, when triggered, sets the gain-term of the auto-nulling algorithm to zero and begins recording the scan data. The sample passes through the aperture, exiting on the other side and triggering a further photo-diode which registers that the scan is complete. The total scan time from start photo-diode to finish photo-diode is approximately 3 seconds. The total time for the sample to pass through the aperture is approximately 1.5 seconds. After scanning, the recorded data is postprocessed using the method described below to obtain a single complex result at each frequency for the sample.

\subsection{Post-processing}

The system returns the complex components of the receive signal for the target frequencies as the sample passes through. Each frequency component gives a typical response profile as it passes through the gradiometer coil assembly around the aperture.

We wish to extract a single complex component from this profile for each of the target frequencies. A match-filter is used to extract this value. The matchfilter is a best-fit of some known, noise-less, ideal profile to the measured data. This known profile is obtained from either a $300 \mathrm{ml}$ glass sample jar - as described in section 4.1.1- containing $0.17 \mathrm{~S} / \mathrm{m}$ saline solution or a $57 \mathrm{~mm}$ PVC sphere containing a $5 \mathrm{~S} / \mathrm{m}$ saline solution. Both targets produce substantially more signal than noise and, for our purposes, can be assumed ideal, i.e. noiseless.

The choice of these two known signals depends on the shape of the test sample. All fruit and vegetable samples used the $5 \mathrm{~S} / \mathrm{m}$ sphere whereas the liquid measurements used the $0.17 \mathrm{~S} / \mathrm{m}$ jar. The known profiles are separately correlated with the real and imaginary components of the measured profiles. The peak on each of the correlated plots, both real and imaginary, are then taken to obtain a single complex value for the frequency component associated with that profile. 
Table 1. Test samples and their properties

\begin{tabular}{lcccccc} 
Sample & Desig. & $\mathrm{h}(\mathrm{mm})$ & $\mathrm{D}_{1}(\mathrm{~mm})$ & $\mathrm{D}_{2}(\mathrm{~mm})$ & Vol. $\left(\mathrm{cm}^{3}\right)$ & Temp. $\left({ }^{\circ} \mathrm{C}\right)$ \\
\hline Granny & $\mathrm{A}$ & 65 & 70 & 68 & 180 & 15.5 \\
Smith Apple & $\mathrm{B}$ & 66 & 74 & 72 & 220 & 15.2 \\
& $\mathrm{C}$ & 67 & 69 & 67 & 190 & 16.2 \\
Gala Apple & $\mathrm{A}$ & 60 & 72 & 66 & 160 & 22.4 \\
& $\mathrm{~B}$ & 62 & 68 & 65 & 160 & 22.4 \\
White Flesh & $\mathrm{C}$ & 60 & 67 & 65 & 150 & 22.5 \\
Nectarines & $\mathrm{A}$ & 56 & 64 & 63 & 120 & 19.6 \\
& $\mathrm{~B}$ & 58 & 64 & 63 & 110 & 20.3 \\
Tomatoes & $\mathrm{C}$ & 56 & 64 & 62 & 110 & 19.9 \\
& $\mathrm{~A}$ & 49 & 61 & 60 & 100 & 22.1 \\
\multirow{5}{*}{ Oranges } & $\mathrm{B}$ & 51 & 64 & 63 & 120 & 22.8 \\
& $\mathrm{C}$ & 48 & 57 & 55 & 80 & 23.0 \\
\multirow{5}{*}{ Pear } & $\mathrm{A}$ & 58 & 60 & 59 & 100 & 24.9 \\
& $\mathrm{~B}$ & 55 & 62 & 61 & 110 & 25.0 \\
\multirow{5}{*}{ Kiwi } & $\mathrm{C}$ & 60 & 63 & 63 & 130 & 25.2 \\
& $\mathrm{~A}$ & 160 & 58 & 59 & 160 & 24.2 \\
\multirow{5}{*}{ Potato } & $\mathrm{B}$ & 160 & 63 & 62 & 160 & 24.3 \\
& $\mathrm{C}$ & 119 & 64 & 61 & 160 & 24.5 \\
& $\mathrm{~A}$ & 65 & 54 & 51 & 100 & 23.7 \\
& $\mathrm{~B}$ & 64 & 55 & 48 & 100 & 23.9 \\
& $\mathrm{C}$ & 69 & 52 & 50 & 100 & 24.0 \\
& $\mathrm{~A}$ & 60 & 89 & 81 & 220 & 24.1 \\
& $\mathrm{~B}$ & 59 & 90 & 83 & 230 & 24.1 \\
& $\mathrm{C}$ & 61 & 84 & 75 & 210 & 24.2
\end{tabular}

\subsection{System calibration}

The system is calibrated using a small cylindrical piece of ferrite (material 4B1, Ferroxcube), $1.8 \mathrm{~mm}$ diameter by $4.2 \mathrm{~mm}$ length. The ferrite-piece is scanned as if it were a sample; that is, using the method described in section 4.2. The scan-time is 1.5 seconds.

The ferrite is assumed to be purely reactive and to have a flat response across the system bandwidth. The ferrite-piece is passed through the point of maximum sensitivity with its central axis in line with the axis of the archway. The system is calibrated by dividing the measured complex post-processed frequency component by the matching complex postprocessed frequency component of the ferrite response, i.e. the returned complex frequency response are phase and magnitude adjusted such that the ferrite response yields +1 on the real axis at the target frequency components. This calibration makes the system output equivalent to equation (7) with the real component a function of permittivity and the imaginary component a function of conductivity.

The suitability of the calibration piece is tested using a high salinity $(>4 \mathrm{~S} / \mathrm{m})$ PVC sphere (as described in section 4.1.1). The expected response of the sphere is a purely imaginary response with only a small real response due to susceptibility of the solution. Phase error after calibration at $2.5 \mathrm{MHz}$ is less than

$0.45^{\circ}$ indicating that phase-errors due to complex permeability of the ferrite at higher frequencies are not significant.

\subsection{Correction for size difference}

A correction function is used to adjust the conductivity for size, specifically radii, of the test samples in table 1 in order to obtain true conductivity values $\sigma$ for each sample. The function is based on the radial relation for a conducting sphere defined in equation (7) combined with the relative conductivity measurement of a known spherical reference sample. The function is as follows:

$\sigma=\frac{a_{r e f}^{5} \sigma_{r e f}}{a^{5} \Sigma_{r e f}} \Sigma$

where $a_{\text {ref }}$ is the radius, $\sigma_{\text {ref }}$ is the conductivity and $\Sigma_{r e f}$ the measured relative conductivity of the reference sample. The terms $a$ and $\Sigma$ are the approximate radius and measured relative conductivity of the test sample respectively.

The function is based on the $a^{5}$ relation implied by equation (7). We briefly review the validity of this relation for our specific coil geometry using electromagnetic field simulations. The MIS system is modelled using Maxwell software (Ansys, USA). A set of spheres of different radii and conductivities were simulated. The chosen range of values (dimensions 


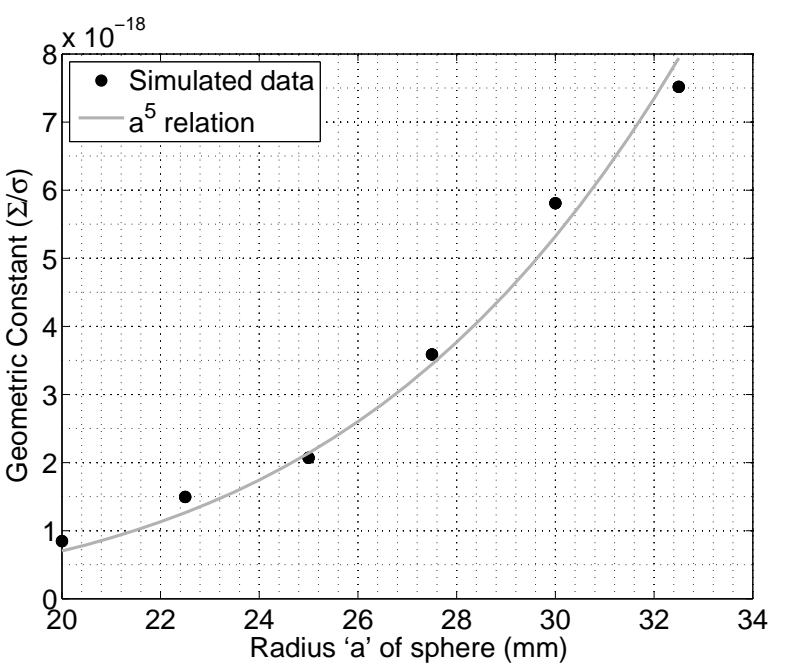

Figure 6. Simulated geometric constant $(\Sigma / \sigma)$ with respect to radius $a$ of a conductive sphere.

and conductivity) were similar to the agricultural produce which we are testing. The external aluminium enclosure is treated as a perfect electrical conductor with no end caps and the size of the model was typically of $100 \mathrm{k}$ tetrahedral elements. The results from the simulations are shown in figure 6. The $a^{5}$ relation is shown as the solid line. Clearly, the relation fits relatively closely with the modelled data over the range of interest. This is despite the sensor head configuration not meeting all the assumptions posed in section 2 , for instance, the assumption of a homogeneous magnetic field across the sample.

\section{Results and discussion}

\subsection{System validation}

In the first test, we compare the measured relative conductivity $\Sigma$, as defined in equation (8), against the known conductivity of a set of saline sample jars. The saline sample jars are passed through the sensorhead. Each jar is passed through the head three times before moving to the next one in a sequence running from lowest to highest conductivity. This sequence is repeated eight times leading to a total of twenty four scans (eight sequential runs with three scans per run). After completion of the test, the saline jars were reopened and measured for conductivity and temperature. All saline solutions were between 23.4$24.0^{\circ} \mathrm{C}$ when conductivity measurements were taken.

The mean of the measured relative conductivity for each sample and the standard deviation about the mean are shown in figure 7. The results show relatively flat spectra for each of the saline samples. This is the expected response. A slight negative gradient is noted. This could be attributed to an imperfectly flat response

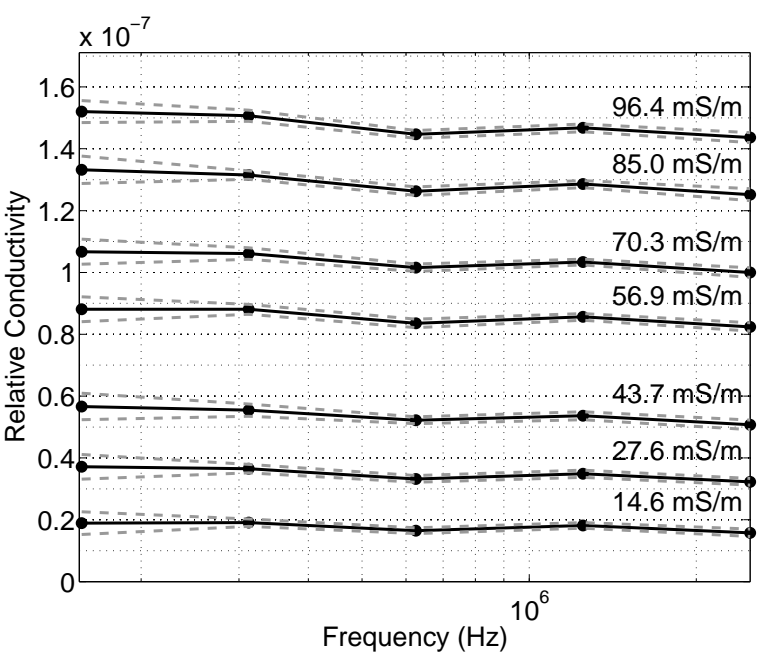

Figure 7. Relative conductivity of saline jars. $(-)=$ mean value, (- -) standard deviation, $\mathrm{N}=24$.

from the calibration ferrite. The standard deviation about each sample appear close to their respective mean values, particularly at the highest frequencies, indicating good consistency and repeatability over the short-term time interval of the experiment. The standard deviation is relatively consistent across the bandwidth with the exception of the lowest frequency which is markedly worse.

Figure 8 shows the signal-to-noise ratio for each frequency for a $56.9 \mathrm{mS} / \mathrm{m}$ sample jar. Each line is a single measurement of the jar, with twenty-four measurements in total. The noise is measured at the end of each sequential-run and is post-processed in the same way. The thick line shows the lower-bound or worst-case signal-to-noise ratio. The lowest frequency shows the worst SNR at $18 \mathrm{~dB}$. This is commensurate with our previous measurements showing widest standard-deviation at the lowest frequency. The highest SNR is $30 \mathrm{~dB}$ at $625 \mathrm{kHz}$ after which a small loss in SNR is observed. This is caused by attenuation in the transmission-line transformer network (at the input to the receive amplifier) when the coil impedance is high.

Comparison between the relative and absolute conductivities are shown in figure 9. The comparison shows broadly linear proportion between the relative and known conductivities. There is some variation, notably between $43.7 \mathrm{mS} / \mathrm{m}$ and $56.9 \mathrm{mS} / \mathrm{m}$, which appears to vary from the established trend. A lineof-best-fit is obtained using least-squares method with $R^{2}=0.9978$.

The long-term stability of the system was investigated by repeated scanning of the ferrite calibration piece over a period of eleven days. The percentage variation in magnitude and phase variation in degrees from the overall mean are shown in figure 


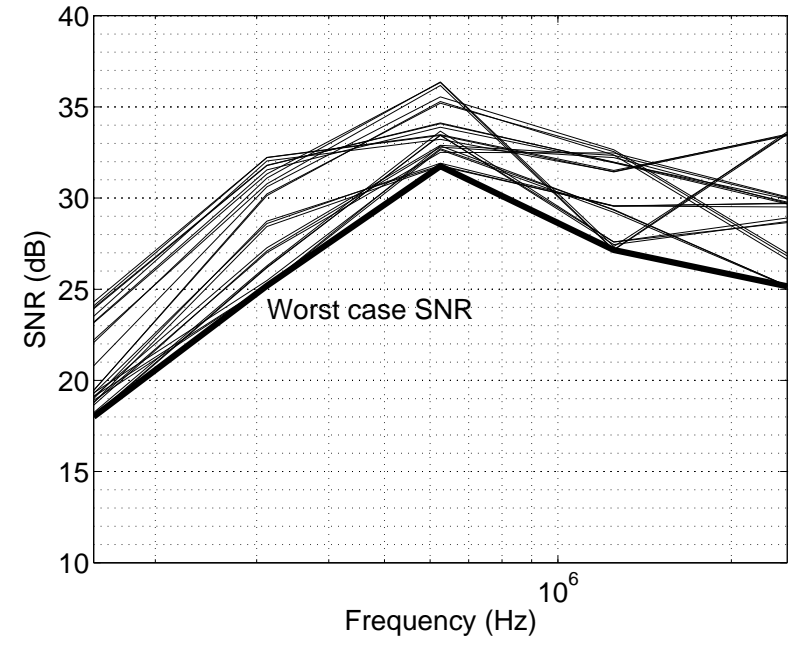

Figure 8. Signal-to-Noise ratio for $56.9 \mathrm{mS} / \mathrm{m}$ Saline jar.

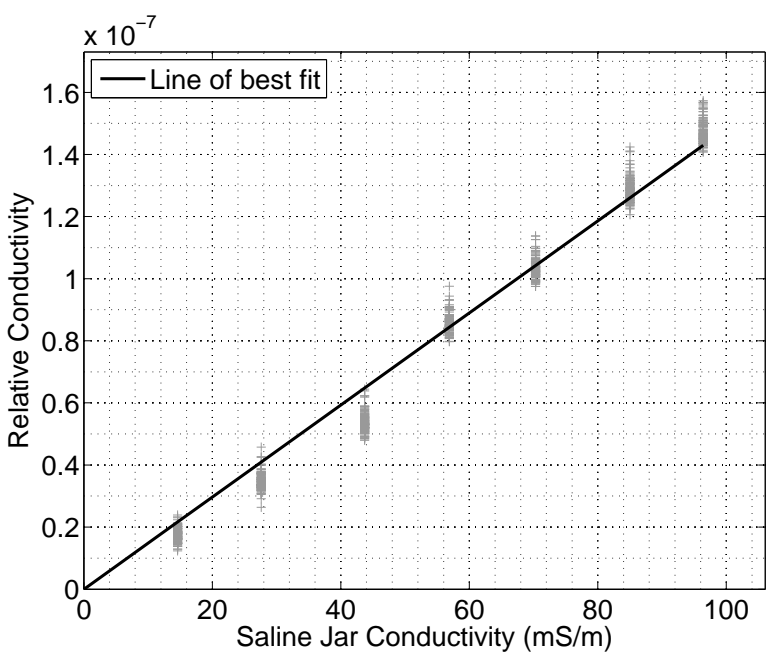

Figure 9. Relative Conductivity vs. Conductivity of Saline Jars.

10. The results show the system is stable over this interval, with no marked drift of the measurements in any direction. The magnitude variation has a standard deviation of $0.93 \%$ and the phase variation $0.46^{\circ}$

\subsection{Yeast suspensions}

Figure 11 shows the conductivity spectra of yeast suspensions of four different concentrations. The results show an upward curvature around $312 \mathrm{kHz}$ to $625 \mathrm{kHz}$ indicating that this point of the spectra is at the initiation of the dispersion. This agrees with Barai et al. 38 and Bragós et al. 29, where the beginning of the dispersion was found over a similar bandwidth and the end of the dispersion found at least a frequency-decade higher.

The degree of curvature appears to broadly correlate with the concentration of the solution -

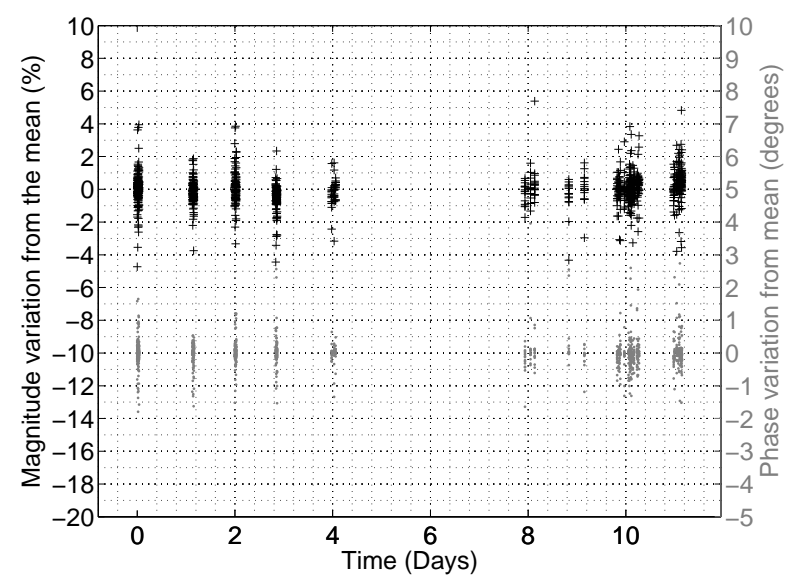

Figure 10. Magnitude and phase variation when measuring ferrite calibration piece over several days.

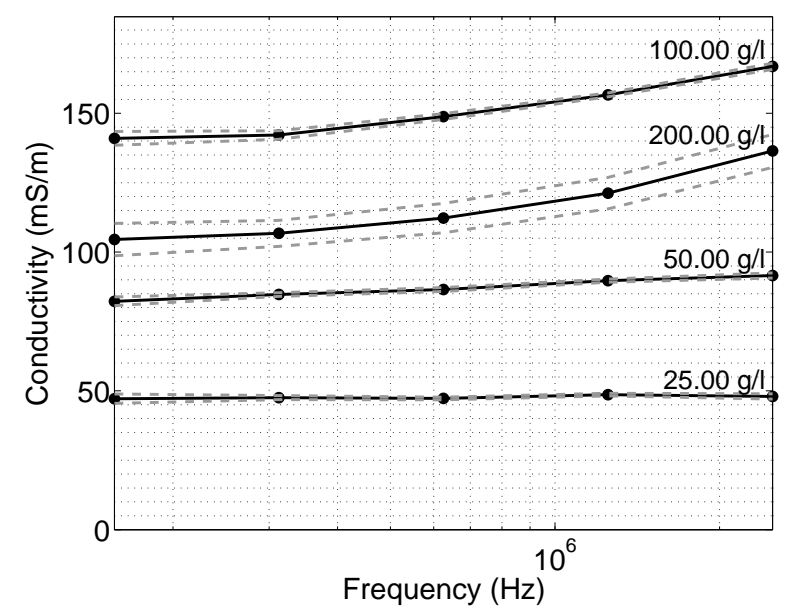

Figure 11. Conductivity spectra of yeast samples.

with higher concentrations featuring more noticeable curvature than the lower concentrations. The rate of curvature was not as significant as shown in Barai et al. [38. The change in conductivity from 156 $\mathrm{kHz}$ to $2.5 \mathrm{MHz}$ was found to be around $50 \%$ higher than this study. Furthermore, a ratio of highto-low frequencies cannot in this case be used as a predictor of concentration. Typically, the highfrequency conductivity should be at or near the top of the dispersion for the predictor to be accurate, whereas our measurements are near the bottom of the dispersion. This would not result in a very sensitive prediction algorithm using ratios.

Good repeatability of the measurements is shown for the yeast samples. Standard deviations are less than $2.5 \mathrm{mS} / \mathrm{m}$ except for the highest concentration sample $(200 \mathrm{~g} / \mathrm{l})$ which showed a much larger standard deviation than the other concentrations. This could be due to the rapid changes in the suspension occurring while under test. The highest concentration was the 
first sample to be tested and there was considerable production of carbon dioxide at the beginning. Large bubbles appeared in the sample jar while testing and the lid visibly bulged due to the pressure. These rapid changes most likely influenced the measurements.

Further, the variation about the mean on the conductivity spectra was not Gaussian, but rather drifted in conductivity with time. The curvature of the spectra however, remained mostly the same. The affects of dynamic active processes were not evident in the lower concentrations derived by dilution from this first sample.

\subsection{Food samples}

The conductivity spectra of several samples of agricultural produce are shown in figures 12 and 13 Figure 12 shows the spectra for two varieties of apples - granny smith and gala. Both varieties have similar conductivities ranging from $40-110 \mathrm{mS} / \mathrm{m}$. For the granny smith apples, sample $\mathrm{C}$ gives the largest conductivity followed by samples A then B. This is in matching order of the two diameters $D_{1}$ and $D_{2}$ for the apples (see table 1) - apple $\mathrm{C}$ having the smallest $\mathrm{D}_{1}$ and $\mathrm{D}_{2}$ followed by $\mathrm{A}$ then $\mathrm{B}$. This is also the case with the gala apples. This indicates that some of the difference between the two samples due to size still remains despite the use of the corrective function.

The corrective equation assumes that the test object is spherical. The apples are not perfectly spherical and this contradiction could account for some of the residual relation to size. Moreover, the internal composition of the fruit, such as the shape and size of the core, and the fruits physicochemical properties may also have an effect.

Figure 13 shows the spectral response of several different fruits and vegetables; including white flesh nectarines, salad tomatoes, oranges, conference pears, kiwis and baked potatoes. Conductivities range from $50 \mathrm{mS} / \mathrm{m}$ to $550 \mathrm{mS} / \mathrm{m}$. Kiwis appear to have the highest overall conductivity. However, for some of the samples - specifically, kiwis, potatoes and pears - caution should be applied to absolute conductivity values. The corrective function assumes perfect spheres which is not true for these samples. Using the $\mathrm{D}_{1}$ diameter for the corrective function will, in these cases, result in poor conductivity correction.

The consistency of the results is promising in both figure 12 and 13 with tight standard deviations about the mean for all samples. The standard deviation at lower-frequencies is in general larger as would be expected for the lower conductivities. The deviations are also higher for the smaller produce such as kiwis and pears. Again, this is anticipated and predicted by the background theory in section 2 (eq. 7).

The spectra of the apple varieties in figure 12 appear to flatten at higher frequencies. Tomatoes and pears in figure 13 show a similar trend. This indicates that the measured bandwidth is at or near the top of the dispersion for these samples. This is not the case for potato which shows a close to linear increase in conductivity with respect to frequency. This indicates that the bandwidth is within the middle-linear portion of the dispersion. Nectarines, oranges and kiwis are more ambiguous and would require a larger bandwidth or a higher resolution spectra to delineate the part of the dispersion with certainty.

The point along the dispersion of the range 156 $\mathrm{kHz}$ to $2.5 \mathrm{MHz}$ for tomatoes broadly agree with the results in Barai et al. 38. Absolute conductivities across the spectra were also found to be similar to Barai et al., with differences of less than $50 \mathrm{mS} / \mathrm{m}$ from each other.

Similarly, the dispersion shape of the potatoes is in broad agreement with the shape presented in Scharfetter et al. 45] and Barai et al. 38 although Barai et al. do show some flattening of the dispersion at $2.5 \mathrm{MHz}$ which is not evident in figure 13:. The differences in absolute conductivity were more significant for potatoes. Differences up to $150 \mathrm{mS} / \mathrm{m}$ were found when compared to Barai et al. 38. This again could be due to poor size correction of potatoes stemming from poor adherence to the spherical assumption used in the corrective function in equation (11). Absolutes conductivities were not presented in Scharfetter et al. 45 .

Published work on nectarines, such as Harker \& Dunlop [18 and Harker \& Maindonald 24, show the initiation of the dispersion occurring between $1 \mathrm{kHz}$ and $100 \mathrm{kHz}$ and a linear region occurring between $10 \mathrm{kHz}$ and $1 \mathrm{MHz}$ when converted into conductivity plots. Our own measurements, shown in figure [13 appear to agree over the range the bandwidths overlap. The bandwidth of the MIS system does not extend lowenough to confirm the initiation of the dispersion.

5.3.1. Temperature variation: We consider the effect of temperature of the sample on the MIS measurements. Figure 14 shows the percentage change in conductivity of gala apples, white flesh nectarines, oranges and potatoes with respect to a change in temperature from some reference value. Lines-of-bestfit are shown for the latter four frequencies. The first frequency - $156 \mathrm{kHz}$ - had poor $R^{2}$ value for all the samples (less than 0.7) so are excluded. The gradient of the line-of-best-fit $\alpha$ is termed the temperature correction coefficient and is defined according to the standard linear temperature correction formula,

$100\left(\frac{\sigma_{\text {meas }}}{\sigma_{\text {ref }}}-1\right)=\alpha \Delta T=\alpha\left(T_{\text {meas }}-T_{\text {ref }}\right)$ 


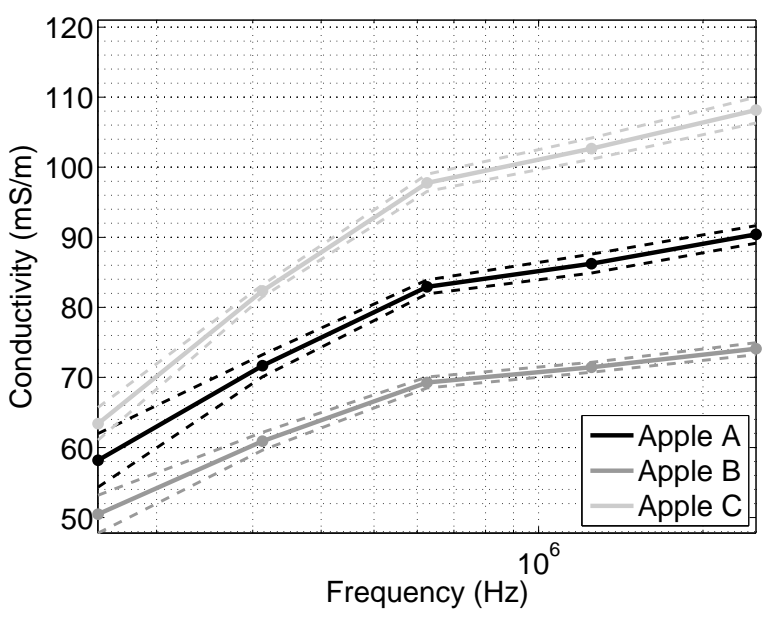

(a)

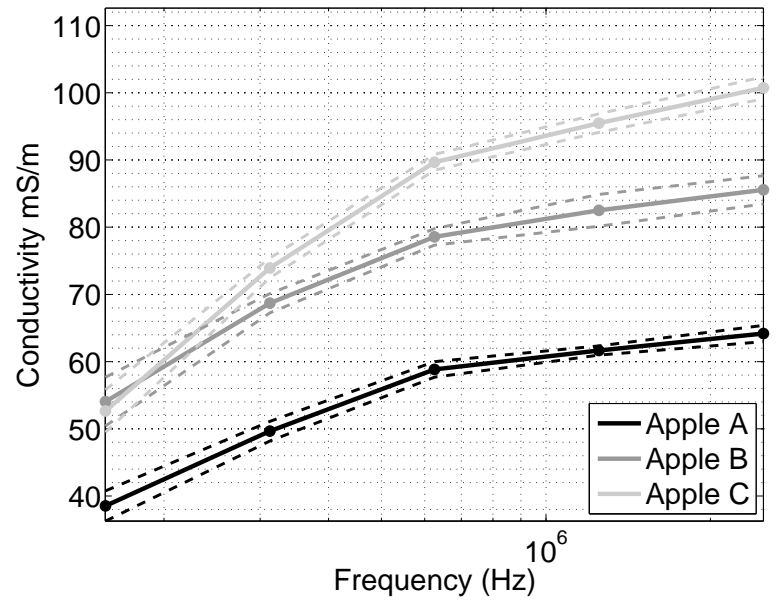

(b)

Figure 12. Conductivity spectra of Apples samples. a) Granny Smith, b) Gala. (-) = mean value, (- -) standard deviation, N=9 per sample.

where $\sigma_{\text {meas }}$ and $T_{\text {meas }}$ are the measured conductivity and temperature respectively, and $\sigma_{r e f}$ and $T_{r e f}$ are the conductivity and temperature at some reference point. The reference point for the samples were 22.6 ${ }^{\circ} \mathrm{C}$ for gala apples, $23.6{ }^{\circ} \mathrm{C}$ for nectarines, $25.0^{\circ} \mathrm{C}$ for oranges and $24.2{ }^{\circ} \mathrm{C}$ for potatoes.

The lines-of-best-fit have good $R^{2}$ values for the highest three frequencies $-625 \mathrm{kHz}$ to $2.5 \mathrm{MHz}$ - greater than 0.9 . For $312 \mathrm{kHz}$, only the results for gala apples have $R^{2}$ greater than $0.9\left(R^{2}=0.92\right)$. A temperature correction coefficient $\alpha$ is calculated for each line-ofbest-fit. The ranges for each sample are as follows: 1.72-2.16 \% $/{ }^{\circ} \mathrm{C}$ for gala apples, $1.02-1.98 \% /{ }^{\circ} \mathrm{C}$ for nectarines, $1.37-1.88 \% /{ }^{\circ} \mathrm{C}$ for oranges and $0.3-1.51$ $\% /{ }^{\circ} \mathrm{C}$ for potatoes.

The size of the range of temperature correction coefficients is surprising, and could have significant implications on the practical use of bio-impedance measurement. In the introduction, we presented past work which used the ratio of high-to-low frequencies as a metric. The assumption is that by using the ratio, we can divide-out external effects such as size, pressure, temperature, etc, which affect all frequencies equally, but retain the effect of the cell-membrane which generally affects the lower frequencies. The variation in the temperature correction coefficient indicates that temperature may not affect the conductivity equally over the spectra and that impedance ratios may retain the consequences of temperature variation in the samples. To the author's knowledge, no previous work has been published which explicitly investigates the temperature correction coefficients for conductivity measurement of agricultural produce. Thus, these results have yet to be replicated elsewhere.

\section{Conclusion}

A magnetic induction spectroscopy system is described which can be used to obtain bio-impedance conductivity spectra of liquid and solid biological samples over a frequency range between $156 \mathrm{kHz}$ to $2.5 \mathrm{MHz}$. The key features of the system are its ruggedness and speed of operation, making it well suited to industrial environments with high-throughput sorting and production lines. The inherent pragmatism and fitness for use at industrial-scale marks a significant advance over existing state-of-the-art in bio-impedance spectroscopy.

The system functioned well in a series of performance tests, giving sensitive and repeatable measurements linearly-correlated with a range of lowconductivity saline targets. Long-term stability tests using a ferrite calibration piece showed only small variations in magnitude and phase over time and no overall drift.

A set of yeast suspensions of differing concentrations and a range of agricultural produce were tested using the MIS system. The system was sufficiently sensitive to obtain consistent conductivity dispersion curves for each of the test samples. Results compared well with existing electrical impedance and magnetic impedance spectroscopic measurements in the literature. These results demonstrate that the MIS system is well-suited to bio-impedance spectroscopy applications in the food industry.

\section{Acknowledgments}

This research was conducted as part of the Fruitgrading Project, "A low cost sorting solution for the fruit sector based on the evaluation of internal fruit quality". 


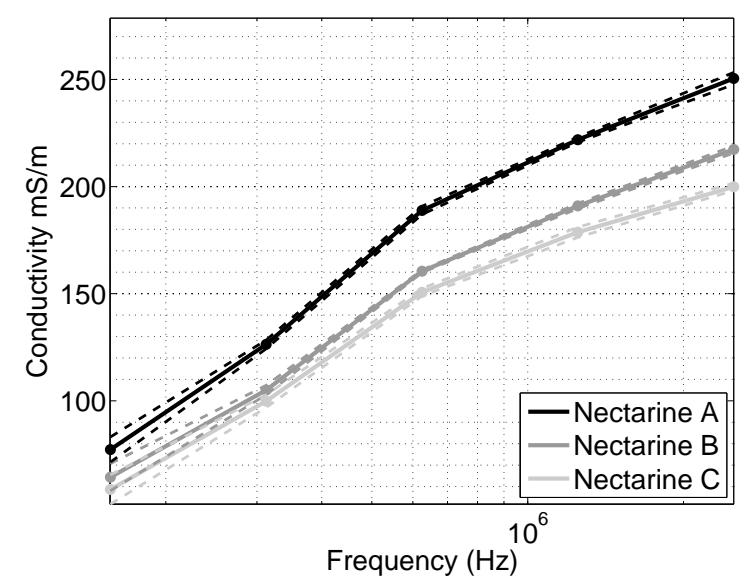

(a)

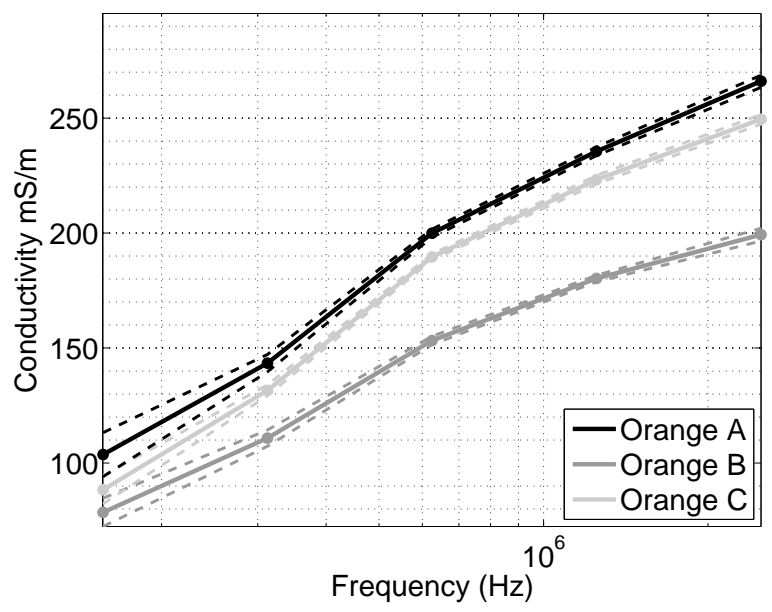

(c)

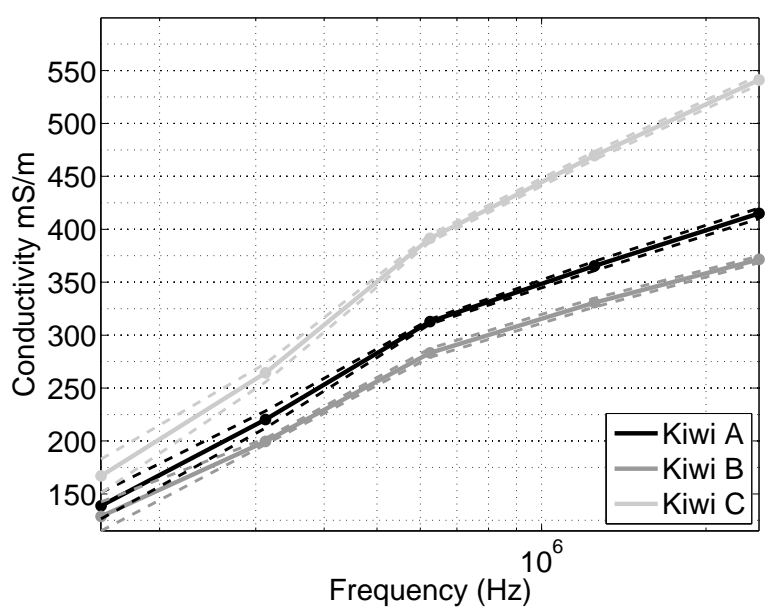

(e)

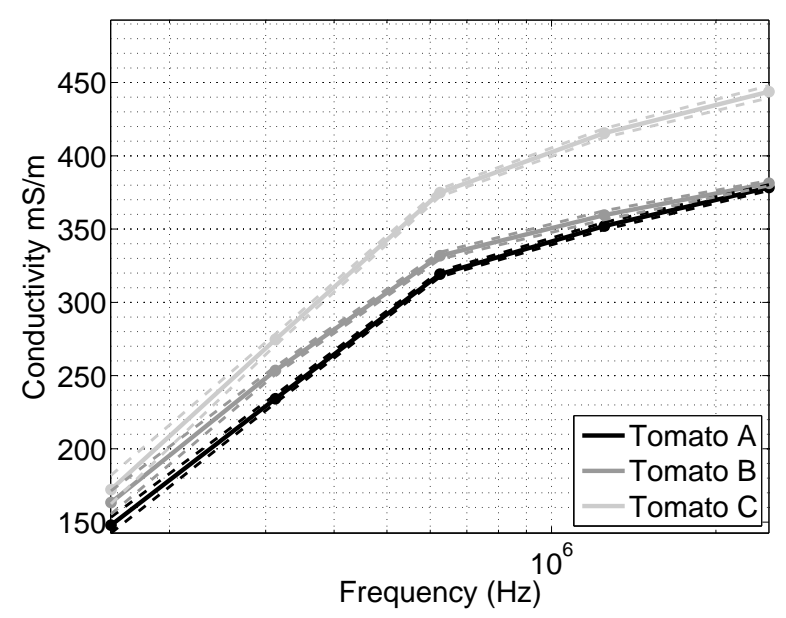

(b)

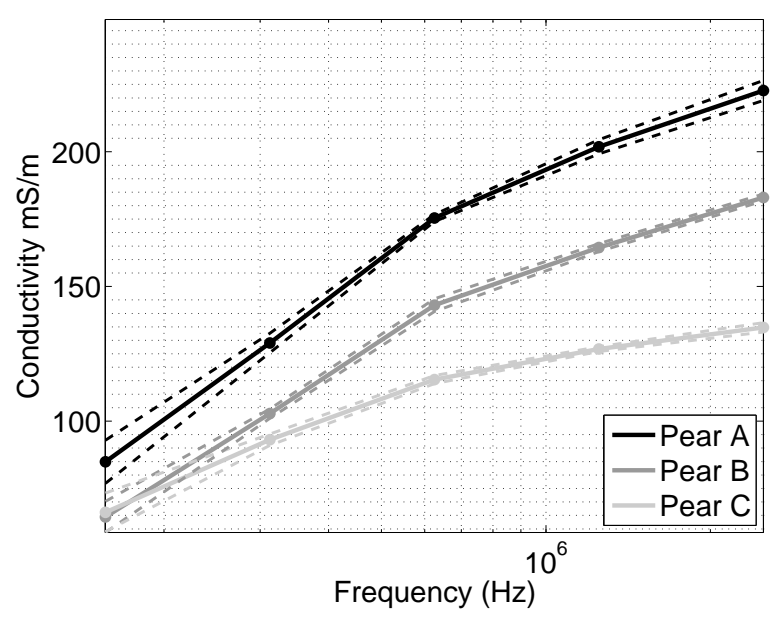

(d)

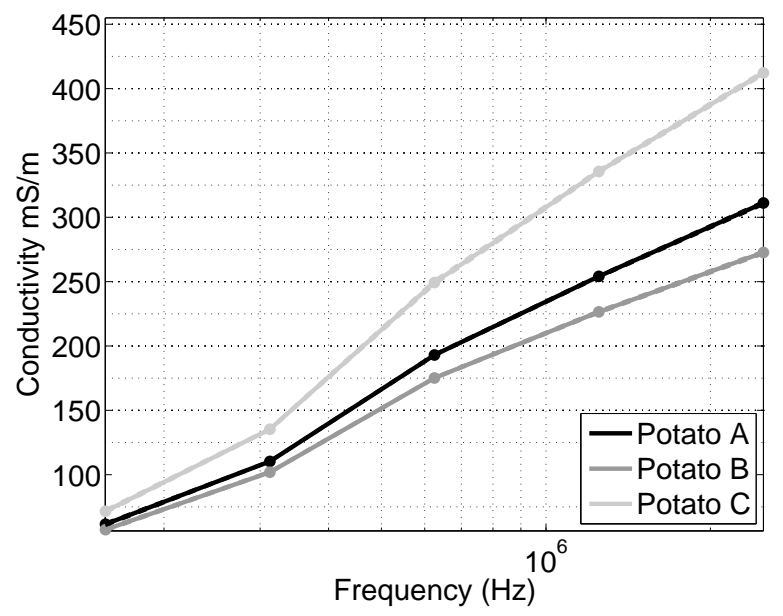

(f)

Figure 13. Conductivity spectra of different agricultural produce. a) White flesh nectarines, b) Salad tomatoes, c) Oranges, d) Conference pears, e) kiwis, f) Baked potatoes. $(-)=$ mean value, (- -) standard deviation, $\mathrm{N}=9$ per sample. 


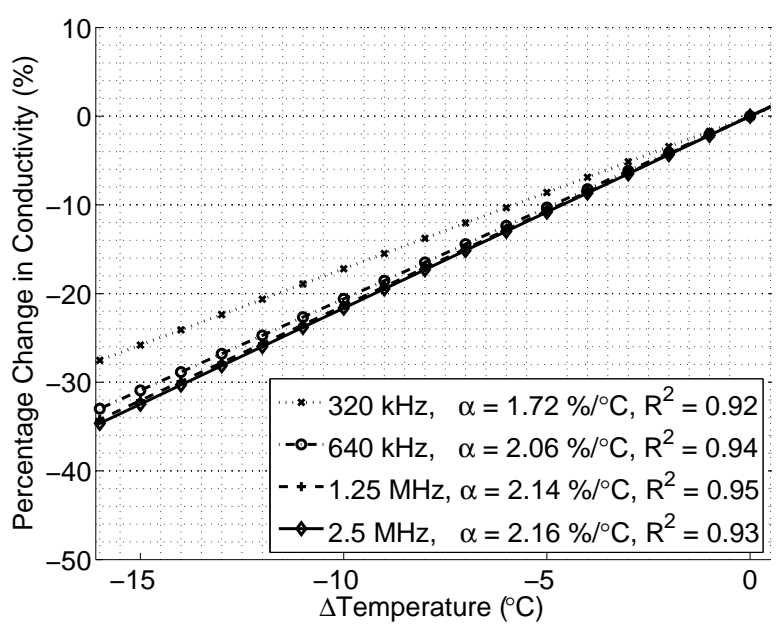

(a)

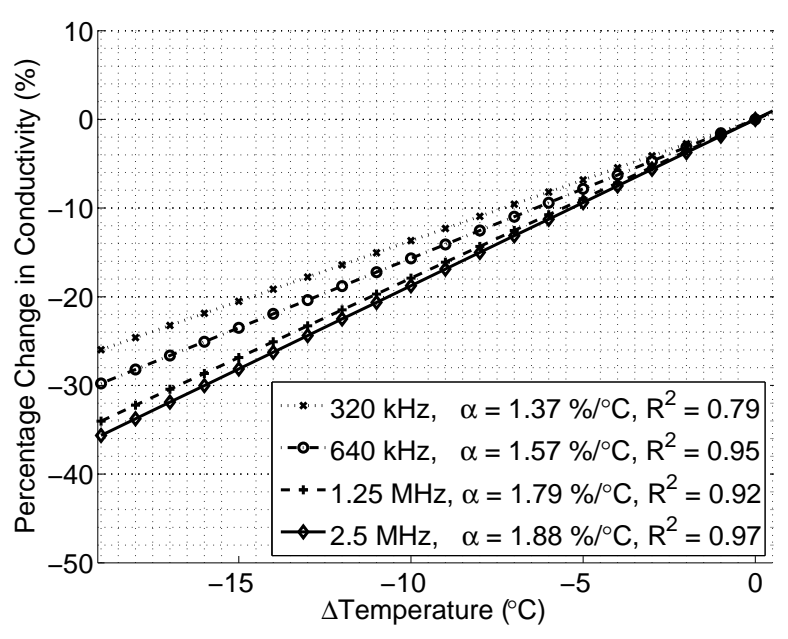

(c)

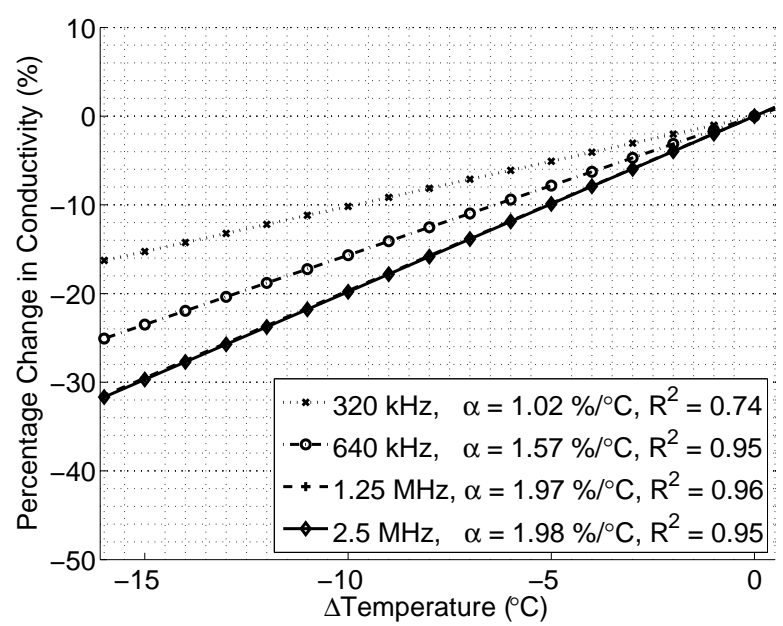

(b)

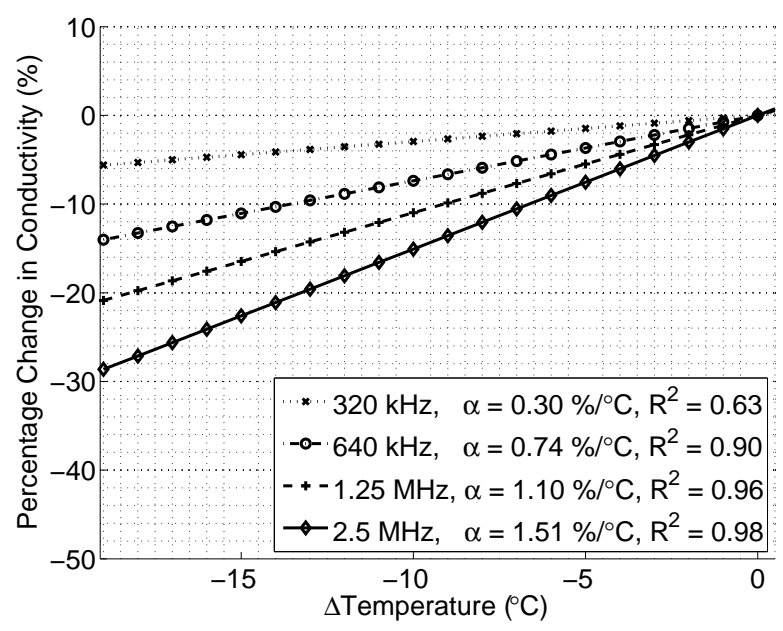

(d)

Figure 14. Lines of best fit for change in conductivity with respect to a change in temperature, a) Gala Apple, b) White flesh Nectarine, c) Orange, d) Potato

The project is funded by the European Union's Seventh Framework Programme for research, technological development and demonstration under grant agreement no 286737.

\section{References}

[1] C. Gabriel, S. Gabriel, and E. Corthout, "The dielectric properties of biological tissues: I. literature survey," Physics in Medicine and Biology, vol. 41, no. 11, p. 2231, 1996.

[2] U. Pliquett, M. Altmann, F. Pliquett, and L. Schberlein, "Pya parameter for meat quality," Meat Science, vol. 65, no. 4, pp. 1429 - 1437, 2003.

[3] M. Y. Jaffrin and H. Morel, "Body fluid volumes measurements by impedance: A review of bioimpedance spectroscopy (bis) and bioimpedance analysis (bia) methods," Medical Engineering 86 Physics, vol. 30, no. 10, pp. 1257 - 1269, 2008.

[4] Z. Zakaria, R. A. Rahim, M. S. B. Mansor, S. Yaacob,
N. M. N. Ayob, S. Z. M. Muji, M. H. F. Rahiman, and S. M. K. S. Aman, "Advancements in transmitters and sensors for biological tissue imaging in magnetic induction tomography," Sensors, vol. 12, no. 6, pp. 71267156, 2012.

[5] R. Merwa and H. Scharfetter, "Magnetic induction tomography: comparison of the image quality using different types of receivers," Physiological Measurement, vol. 29, no. 6, p. S417, 2008.

[6] E. Callow, "The electrical resistance of muscular tissue and its relation to curing," Special Report, vol. 75, pp. 75-81, 1936.

[7] J.-L. Damez and S. Clerjon, "Quantifying and predicting meat and meat products quality attributes using electromagnetic waves: An overview," Meat Science, vol. 95, no. 4, pp. 879 - 896, 2013.

[8] M. Castro-Giráldez, P. Botella, F. Toldrá, and P. Fito, "Low-frequency dielectric spectrum to determine pork meat quality," Innovative Food Science \& Emerging Technologies, vol. 11, no. 2, pp. 376 - 386, 2010.

[9] M. Oliver, I. Gobantes, J. Arnau, J. Elvira, P. Riu, N. Grbol, and J. M. Monfort, "Evaluation of the 
electrical impedance spectroscopy (eis) equipment for ham meat quality selection," Meat Science, vol. 58, no. 3, pp. $305-312,2001$.

[10] J. Lepetit, P. Sal, R. Favier, and R. Dalle, "Electrical impedance and tenderisation in bovine meat," Meat Science, vol. 60, no. 1, pp. $51-62,2002$.

[11] J.-L. Damez and S. Clerjon, "Meat quality assessment using biophysical methods related to meat structure," Meat Science, vol. 80, no. 1, pp. 132 - 149, 2008.

[12] J.-L. Damez, S. Clerjon, S. Abouelkaram, and J. Lepetit, "Electrical impedance probing of the muscle food anisotropy for meat ageing control," Food Control, vol. 19, no. 10, pp. $931-939,2008$.

[13] C. Byrne, D. Troy, and D. Buckley, "Postmortem changes in muscle electrical properties of bovine $\mathrm{m}$. longissimus dorsi and their relationship to meat quality attributes and ph fall," Meat Science, vol. 54, no. 1, pp. 23 - 34, 2000.

[14] M. Chanet, C. Rivire, and P. Eynard, "Electric impedance spectrometry for the control of manufacturing process of comminuted meat products," Journal of Food Engineering, vol. 42, no. 3, pp. 153 - 159, 1999.

[15] R. Masot, M. Alcaiz, A. Fuentes, F. C. Schmidt, J. M. Barat, L. Gil, D. Baigts, R. Martnez-Mez, and J. Soto, "Design of a low-cost non-destructive system for punctual measurements of salt levels in food products using impedance spectroscopy," Sensors and Actuators A: Physical, vol. 158, no. 2, pp. $217-223,2010$.

[16] A. Rizo, A. Fuentes, I. Fernndez-Segovia, R. Masot, M. Alcaiz, and J. M. Barat, "Development of a new salmon saltingsmoking method and process monitoring by impedance spectroscopy," $\{L W T\}$ - Food Science and Technology, vol. 51, no. 1, pp. $218-224,2013$.

[17] S. Bureau, D. Bertrand, B. Jallais, P. Reling, B. Gouble, C. M. G. C. Renard, B. Dekdouk, L. A. Marsh, M. D. OToole, D. W. Armitage, A. J. Peyton, and J. AlvarezGarcia, "Fruitgrading: Development of a fruit sorting technology based on internal quality parameters," in 16th International Conference on Near Infrared Spectroscopy, Montpellier, France, 2013.

[18] F. Harker and J. Dunlop, "Electrical impedance studies of nectarines during coolstorage and fruit ripening," Postharvest Biology and Technology, vol. 4, no. 12, pp. 125 - 134, 1994.

[19] F. R. Harker and S. K. Forbes, "Ripening and development of chilling injury in persimmon fruit: An electrical impedance study," New Zealand Journal of Crop and Horticultural Science, vol. 25, no. 2, pp. 149-157, 1997.

[20] J. Juansah, I. W. Budiastra, K. Dahlan, and K. B. Seminar, "Electrical properties of garut citrus fruits at low alternating current signal and its correlation with physicochemical properties during maturation," International Journal of Food Properties, vol. 17, no. 7, pp. 1498-1517, 2014.

[21] M. Rehman, B. A. Abu Izneid, M. Z. Abdullah, and M. R. Arshad, "Assessment of quality of fruits using impedance spectroscopy," International Journal of Food Science \& Technology, vol. 46, no. 6, pp. 1303-1309, 2011.

[22] M. Soltani, R. Alimardani, and M. Omid, "Evaluating banana ripening status from measuring dielectric properties," Journal of Food Engineering, vol. 105, no. 4, pp. $625-631,2011$.

[23] R. E. Lill, E. M. O'Donoghue, and G. A. King, Postharvest Physiology of Peaches and Nectarines, pp. 413-452. John Wiley \& Sons, Inc., 1989.

[24] F. R. Harker and J. H. Maindonald, "Ripening of nectarine fruit (changes in the cell wall, vacuole, and membranes detected using electrical impedance measurements)," Plant Physiology, vol. 106, no. 1, pp. 165-171, 1994.

[25] E. Vozáry, P. Lásló, and G. Zsivánovits, "Impedance parameter characterizing apple bruise," Annals of the New York Academy of Sciences, vol. 873, no. 1, pp. 421429, 1999.

[26] P. J. Jackson and F. R. Harker, "Apple bruise detection by electrical impedance measurement," HortScience, vol. 35, no. 1, pp. 104-107, 2000.

[27] C. Justice, A. Brix, D. Freimark, M. Kraume, P. Pfromm, B. Eichenmueller, and P. Czermak, "Process control in cell culture technology using dielectric spectroscopy," Biotechnology Advances, vol. 29, no. 4, pp. 391 - 401, 2011.

[28] J. Carvell and J. Dowd, "On-line measurements and control of viable cell density in cell culture manufacturing processes using radio-frequency impedance," Cytotechnology, vol. 50, no. 1-3, pp. 35-48, 2006.

[29] R. Bragós, X. Gámez, J. Cairó, P. J. Riu, and F. Gòdia, "Biomass monitoring using impedance spectroscopy," Annals of the New York Academy of Sciences, vol. 873, no. 1, pp. 299-305, 1999.

[30] A. Soley, M. Lecina, X. Gámez, J. Cairó, P. Riu, X. Rosell, R. Bragós, and F. Gòdia, "On-line monitoring of yeast cell growth by impedance spectroscopy," Journal of Biotechnology, vol. 118, no. 4, pp. $398-405,2005$.

[31] P. Tibayrenc, L. Preziosi-Belloy, and C. Ghommidh, "Online monitoring of dielectrical properties of yeast cells during a stress-model alcoholic fermentation," Process Biochemistry, vol. 46, no. 1, pp. 193 - 201, 2011.

[32] C. Davey, R. Todd, and J. Barrett, "From concept to market in industrial impedance applications," Annals of the New York Academy of Sciences, vol. 873, no. 1, pp. 239-244, 1999.

[33] Y. Kitamura, K. Toyoda, and B. Park, "Electric impedance spectroscopy for yogurt processing," Food Science and Technology Research, vol. 6, no. 4, pp. 310-313, 2000.

[34] X. Li, K. Toyoda, and I. Ihara, "Coagulation process of soymilk characterized by electrical impedance spectroscopy," Journal of Food Engineering, vol. 105, no. 3, pp. $563-568,2011$.

[35] M. Altmann and U. Pliquett, "Prediction of intramuscular fat by impedance spectroscopy," Meat Science, vol. 72, no. 4 , pp. $666-671,2006$.

[36] A. D. Bauchot, F. Harker, and W. Arnold, "The use of electrical impedance spectroscopy to assess the physiological condition of kiwifruit," Postharvest Biology and Technology, vol. 18, no. 1, pp. 9 - 18, 2000.

[37] U. Pliquett, "Bioimpedance: A review for food processing," Food Engineering Reviews, vol. 2, no. 2, pp. 74-94, 2010.

[38] A. Barai, S. Watson, H. Griffiths, and R. Patz, "Magnetic induction spectroscopy: non-contact measurement of the electrical conductivity spectra of biological samples," Measurement Science and Technology, vol. 23, no. 8, 2012.

[39] F. Euring, W. Russ, W. Wilke, and U. Grupa, "Development of an impedance measurement system for the detection of decay of apples," Procedia Food Science, vol. 1, no. 0 , pp. $1188-1194,2011$. 11th International Congress on Engineering and Food (ICEF11).

[40] S. Gabriel, R. W. Lau, and C. Gabriel, "The dielectric properties of biological tissues: Ii. measurements in the frequency range $10 \mathrm{hz}$ to $20 \mathrm{ghz}, "$ Physics in Medicine and Biology, vol. 41, no. 11, p. 2251, 1996.

[41] S. Gabriel, R. W. Lau, and C. Gabriel, "The dielectric properties of biological tissues: Iii. parametric models for the dielectric spectrum of tissues," Physics in Medicine and Biology, vol. 41, no. 11, p. 2271, 1996.

[42] R. I. Hayden, C. A. Moyse, F. W. Calder, D. P. Crawford, and D. S. Fensom, "Electrical impedance studies on potato and alfalfa tissue," Journal of Experimental Botany, vol. 20, no. 2, pp. 177-200, 1969.

[43] M. I. N. Zhang, D. G. Stout, and J. H. M. Willison, 
"Electrical impedance analysis in plant tissues: Symplasmic resistance and membrane capacitance in the hayden model," Journal of Experimental Botany, vol. 41, no. 3, pp. 371-380, 1990.

[44] C. Bidinosti, E. Chapple, and M. Hayden, "The sphere in a uniform rf fieldrevisited," Concepts in Magnetic Resonance Part B: Magnetic Resonance Engineering, vol. 31B, no. 3, pp. 191-202, 2007.

[45] H. Scharfetter, H. K. Lackner, and J. Rosell, "Magnetic induction tomography: hardware for multi-frequency measurements in biological tissues," Physiological Measurement, vol. 22, no. 1, p. 131, 2001. 